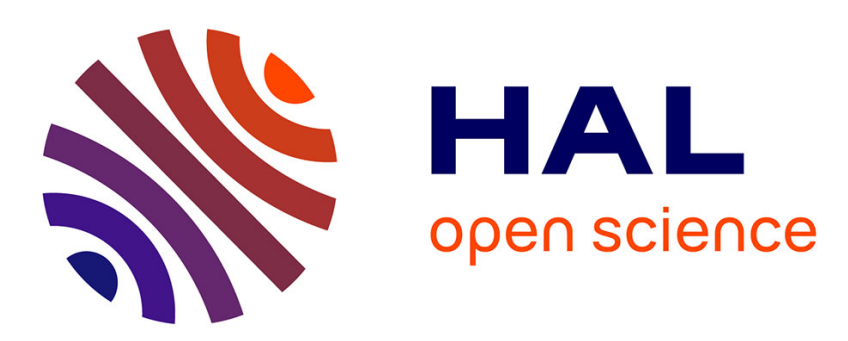

\title{
Prédiction du besoin transfusionnel chez le patient traumatisé hémodynamiquement stable par la mesure du lactate capillaire au déchocage
}

Clotilde Schilte

\section{- To cite this version:}

Clotilde Schilte. Prédiction du besoin transfusionnel chez le patient traumatisé hémodynamiquement stable par la mesure du lactate capillaire au déchocage. Médecine humaine et pathologie. 2013. dumas-00925807

\section{HAL Id: dumas-00925807 https://dumas.ccsd.cnrs.fr/dumas-00925807}

Submitted on 8 Jan 2014

HAL is a multi-disciplinary open access archive for the deposit and dissemination of scientific research documents, whether they are published or not. The documents may come from teaching and research institutions in France or abroad, or from public or private research centers.
L'archive ouverte pluridisciplinaire HAL, est destinée au dépôt et à la diffusion de documents scientifiques de niveau recherche, publiés ou non, émanant des établissements d'enseignement et de recherche français ou étrangers, des laboratoires publics ou privés. 


\section{Université
Joseph Fourier MEDECINE}

\section{SICD1 \\ Bibliothèques \\ de I'UJF et de Grenoble INP}

\section{AVERTISSEMENT}

Ce document est le fruit d'un long travail approuvé par le jury de soutenance et mis à disposition de l'ensemble de la communauté universitaire élargie.

Il n'a pas été réévalué depuis la date de soutenance.

Il est soumis à la propriété intellectuelle de l'auteur. Ceci implique une obligation de citation et de référencement lors de l'utilisation de ce document.

D'autre part, toute contrefaçon, plagiat, reproduction illicite encourt une poursuite pénale.

Contact au SICD1 de Grenoble : thesebum@ujf-grenoble.fr

\section{LIENS}

Code de la Propriété Intellectuelle. articles L 122. 4

Code de la Propriété Intellectuelle. articles L 335.2- L 335.10

http://www.cfcopies.com/V2/leg/leg_droi.php

http://www.culture.gouv.fr/culture/infos-pratiques/droits/protection.htm 
UNIVERSITE JOSEPH FOURIER

FACULTE DE MEDECINE DE GRENOBLE

Année $2013 n^{\circ}$

Prédiction du besoin transfusionnel

chez le patient traumatisé hémodynamiquement stable par la mesure du lactate capillaire au déchocage

\title{
THESE
}

\section{PRESENTEE POUR L'OBTENTION DU}

DOCTORAT EN MEDECINE DIPLOME D'ETAT

Et soutenue publiquement à la Faculté de Médecine de Grenoble

\section{LE VENDREDI 6 DECEMBRE 2013}

\author{
Par \\ SCHILTE Clotilde \\ Née le 21 mai 1984 à Paris $14^{\text {ème }}$ (75)
}

Devant le Jury composé de :

Président du Jury :

Monsieur le Professeur Jean-François Payen de la Garanderie

Membres du Jury :

Monsieur le Professeur Pierre Albaladejo

Monsieur le Docteur Pierre Bouzat

Monsieur le Docteur Marc Vinclair (Directeur de Thèse) 
Doyen de la Faculté de Médecine: Monsieur le Professeur Jean-Paul ROMANET

\begin{tabular}{|c|c|}
\hline ALBALADEJO Pierre & Anesthésiologie réanimation \\
\hline ARVIEUX-BARTHELEMY Catherine & Chirurgie générale \\
\hline BACONNIER Pierre & $\begin{array}{l}\text { Biostatiques, informatique médicale et technologies de } \\
\text { communication }\end{array}$ \\
\hline BAGUET Jean-Philippe & Cardiologie \\
\hline BALOSSO Jacques & Radiothérapie \\
\hline BARRET LUC & Médecine légale et droit de la santé \\
\hline BAUDAIN Philippe & Radiologie et imagerie médicale \\
\hline BEANI Jean-Claude & Dermato-vénéréologie \\
\hline BENHAMOU Pierre Yves & Endocrinologie, diabète et maladies métaboliques \\
\hline BERGER François & Biologie cellulaire \\
\hline BLIN Dominique & Chirurgie thoracique et cardio-vasculaire \\
\hline BONAZ Bruno & Gastro-entérologie, hépatologie, addictologie \\
\hline BOSSON Jean-Luc & $\begin{array}{l}\text { Biostatiques, informatique médicale et technologies de } \\
\text { communication }\end{array}$ \\
\hline BOUGEROL Thierry & Psychiatrie d'adultes \\
\hline BOUILLET Laurence & Médecine interne \\
\hline BRAMBILLA Christian & Pneumologie \\
\hline BRAMBILLA Elisabeth & Anatomie et cytologie pathologiques \\
\hline BRICAULT Ivan & Radiologie et imagerie médicale \\
\hline BRICHON Pierre-Yves & Chirurgie thoracique et cardio-vasculaire \\
\hline CAHN Jean-Yves & Hématologie \\
\hline CARPENTIER Françoise & Thérapeutique, médecine d'urgence \\
\hline CARPENTIER Patrick & Chirurgie vasculaire, médecine vasculaire \\
\hline CESBRON Jean-Yves & Immunologie \\
\hline CHABARDES Stephan & Neurochirurgie \\
\hline CHABRE Olivier & Endocrinologie, diabète et maladies métaboliques \\
\hline CHAFFANJON Philippe & Anatomie \\
\hline CHAVANON Olivier & Chirurgie thoracique et cardio-vasculaire \\
\hline CHIQUET Christophe & Ophtalmologie \\
\hline CHIROSSEL Jean-Paul & Anatomie \\
\hline CINQUIN Philippe & $\begin{array}{l}\text { Biostatiques, informatique médicale et technologies de } \\
\text { communication }\end{array}$ \\
\hline COHEN Olivier & $\begin{array}{l}\text { Biostatiques, informatique médicale et technologies de } \\
\text { communication }\end{array}$ \\
\hline COUTURIER Pascal & Gériatrie et biologie du vieillissement \\
\hline CRACOWSKI Jean-Luc & Pharmacologie fondamentale, pharmacologie clinique \\
\hline DE GAUDEMARIS Régis & Médecine et santé au travail \\
\hline
\end{tabular}


DEBILLON Thierry

DEMATTEIS Maurice

DEMONGEOT Jacques

DESCOTES Jean-Luc

ESTEVE François

FAGRET Daniel

FAUCHERON Jean-Luc

FERRETTI Gilbert

FEUERSTEIN Claude

FONTAINE Eric

FRANCOIS Patrice

GARBAN Frédéric

GAUDIN Philippe

GAVAZZI Gaetan

GAY Emmanuel

GRIFFET Jacques

HALIMI Serge

HENNEBICQ Sylviane

HOFFMANN Pascale

HOMMEL Marc

JOUK Pierre-Simon

JUVIN Robert

KAHANE Philippe

KRACK Paul

KRAINIK Alexandre

LABARERE José

LANTUEJOUL Sylvie

LE BAS Jean-François

LEBEAU Jacques

LECCIA Marie-Thérèse

LEROUX Dominique

LEROY Vincent

LETOUBLON Christian

LEVY Patrick

LUNARDI Joël

MACHECOURT Jacques

MAGNE Jean-Luc

MAITRE Anne

MAURIN Max

MERLOZ Philippe

MORAND Patrice

MORO Elena

MORO-SIBILOT Denis

MOUSSEAU Mireille

MOUTET François

PALOMBI Olivier

PASSAGIA Jean-Guy
Pédiatrie

Addictologie

Biostatiques, informatique médicale et technologies de

communication

Urologie

Biophysique et médecine nucléaire

Biophysique et médecine nucléaire

Chirurgie générale

Radiologie et imagerie médicale

Physiologie

Nutrition

Epidémiologie, économie de la santé et prévention

Hématologie, transfusion

Rhumatologie

Gériatrie et biologie du vieillissement

Neurochirurgie

Chirurgie infantile

Nutrition

Génétique et procréation

Gynécologie obstétrique

Neurologie

Génétique

Rhumatologie

Physiologie

Neurologie

Radiologie et imagerie médicale

Département de veille sanitaire

Anatomie et cytologie pathologiques

Biophysique et médecine nucléaire

Chirurgie maxillo-faciale et stomatologie

Dermato-vénéréologie

Génétique

Gastro-entérologie, hépatologie, addictologie

chirurgie générale

Physiologie

Biochimie et biologie moléculaire

Cardiologie

Chirurgie vasculaire

Médecine et santé au travail

Bactériologie - virologie

Chirurgie orthopédique et traumatologie

Bactériologie - virologie

Neurologie

Pneumologie

Cancérologie

Chirurgie plastique, reconstructrice et esthétique

Anatomie

Anatomie 
PAYEN DE LA GARANDERIE JeanFrançois

PELLOUX Hervé

PEPIN Jean-Louis

PERENNOU Dominique

PERNOD Gilles

PIOLAT Christian

PISON Christophe

PLANTAZ Dominique

POLACK Benoît

PONS Jean-Claude

RAMBEAUD Jacques

REYT Emile

RIGHINI Christian

ROMANET J. Paul

SARAGAGLIA Dominique

SCHMERBER Sébastien

SCHWEBEL Carole

SERGENT Fabrice

SESSA Carmine

STAHL Jean-Paul

STANKE Françoise

TIMSIT Jean-François

TONETTI Jérôme

TOUSSAINT Bertrand

VANZETTO Gérald

VUILLEZ Jean-Philippe

WEIL Georges

ZAOUI Philippe

ZARSKI Jean-Pierre
Anesthésiologie réanimation

Parasitologie et mycologie

Physiologie

Médecine physique et de réadaptation

Médecine vasculaire

Chirurgie infantile

Pneumologie

Pédiatrie

Hématologie

Gynécologie obstétrique

Urologie

Oto-rhino-laryngologie

Oto-rhino-laryngologie

Ophtalmologie

Chirurgie orthopédique et traumatologie

Oto-rhino-laryngologie

Réanimation médicale

Gynécologie obstétrique

Chirurgie vasculaire

Maladies infectieuses, maladies tropicales

Pharmacologie fondamentale

Réanimation

Chirurgie orthopédique et traumatologie

Biochimie et biologie moléculaire

Cardiologie

Biophysique et médecine nucléaire

Epidémiologie, économie de la santé et prévention

Néphrologie

Gastro-entérologie, hépatologie, addictologie 
APTEL Florent

BOISSET Sandrine

BONNETERRE Vincent

BOTTARI Serge

BOUTONNAT Jean

BOUZAT Pierre

BRENIER-PINCHART M.Pierre

BRIOT Raphaël

CALLANAN-WILSON Mary

DERANSART Colin

DETANTE Olivier

DIETERICH Klaus

DUMESTRE-PERARD Chantal

EYSSERIC Hélène

FAURE Julien

GILLOIS Pierre

GRAND Sylvie

GUZUN Rita

LAPORTE François

LARDY Bernard

LARRAT Sylvie

LAUNOIS-ROLLINAT Sandrine

MALLARET Marie-Reine

MAUBON Danièle

MC LEER (FLORIN) Anne

MOREAU-GAUDRY Alexandre

MOUCHET Patrick

PACLET Marie-Hélène

PAYSANT François

PELLETIER Laurent

RAY Pierre

RIALLE Vincent

ROUX-BUISSON Nathalie

SATRE Véronique

STASIA Marie-Josée

TAMISIER Renaud
Ophtalmologie

Agents infectieux

Médecine et santé au travail

Biologie cellulaire

Cytologie et histologie

Réanimation

Parasitologie et mycologie

Thérapeutique, médecine d'urgence

Hématologie, transfusion

Physiologie

Neurologie

Génétique et procréation

Immunologie

Médecine légale et droit de la santé

Biochimie et biologie moléculaire

Biostatiques, informatique médicale et technologies de communication

Radiologie et imagerie médicale

Endocrinologie, diabétologie, nutrition, éducation

Biochimie et biologie moléculaire

Biochimie et biologie moléculaire

Bactériologie, virologie

Physiologie

Epidémiologie, économie de la santé et prévention

Parasitologie et mycologie

Cytologie et histologie

Biostatiques, informatique médicale et technologies de communication

Physiologie

Biochimie et biologie moléculaire

Médecine légale et droit de la santé

Biologie cellulaire

Génétique

Biostatiques, informatique médicale et technologies de

Biochimie, toxicologie et pharmacologie

Génétique

Biochimie et biologie moléculaire

Physiologie 
REMERCIEMENTS 


\section{Au président du Jury,}

Monsieur le professeur Jean François Payen,

Recevez ici tous mes remerciements pour votre encadrement au cours de ces 5 années passées au sein du pôle anesthésie réanimation. Vous avez à cœur, en plus de l'enseignement clinique, de faire découvrir et participer aux projets de recherche les internes d'anesthésie réanimation, nous faisant ainsi découvrir une autre façon d'exercer notre métier. J'ai apprécié votre qualité d'écoute lors des quelques années passées comme représentante des internes. Je vous remercie également de m'avoir fait confiance pour cette année recherche passée au laboratoire de neuroscience, cela m’a permis de découvrir un autre univers, ce qui est toujours enrichissant intellectuellement.

\section{Aux membres du jury,}

Monsieur le professeur Pierre Albaladejo,

Merci d'avoir accepté de juger ce travail. Soyez aussi remercié pour vos critiques constructives lors de l'élaboration de ce travail et votre aide quant à l'expression des résultats. La clarté de vos messages et de vos exposés sont un exemple pour nous tous, il me reste un long chemin à parcourir pour y parvenir.

Monsieur le docteur Pierre Bouzat, maître de conférences,

Merci pour ton compagnonnage aussi bien en clinique lors de mes premières gardes en réanimation chirurgicale, qu'en recherche lors de la rédaction de mon dossier d'année recherche. Tu m'as permis, grâce à l'obtention de la bourse de passer une année au laboratoire avec pour seule préoccupation la découverte de ce milieu bien étrange qu'est la recherche fondamentale. Tu restes malgré ton ascension professorale toujours accessible et réactif pour donner un conseil.

Monsieur le docteur Marc Vinclair, directeur de thèse

Merci de m'avoir fait confiance pour réaliser ce travail. Tu as été l'instigateur de ce travail et tu as accepté que j'en fasse ma thèse. Merci pour ta grande disponibilité et ton investissement dans la réalisation de ce travail. Ta motivation et ton soutien ont été un réel moteur pour moi pour faire aboutir ce travail. J'ai aussi apprécié travailler avec toi au bloc et en réa pour ta préoccupation permanente de l'intérêt du patient et de leur famille 


\section{A ma famille,}

Elle est formidable ma famille, je ne l'ai pas choisie, mais je ne l'échangerais contre rien au monde.

Maman, tu m'as appris l'importance du travail bien fait, je suis encore loin de tes exigences, mais je m'améliore. C'est grâce à toi si j'ai fait médecine, c'est toi qui m’a donné l'idée et qui m’a ensuite permis de réussir le concours. Ton investissement pour les autres, tes élèves et maintenant au sein de ton association ont toujours été un exemple pour moi.

Papa, pas facile pour toi de suivre ces longues études, mais ça y est j'ai bientôt fini. Tes réussites, professionnelle et associative, sont pour moi des exemples à suivre. Tu as toujours su trouver ou apporter une solution face aux difficultés rencontrées sans jamais négliger ta famille.

Delphine, je ne peux penser à ces longues heures passées devant mon bureau sans voir ta présence plus ou moins patiente à mes côtés. Tu as été un moteur extraordinaire pour m'inciter à me dépasser et ne pas céder à la facilité. Merci pour ton amour inconditionnel. Tu me manques.

Clémentine, j'ai fait médecine pour ne pas faire comme toi, trop peur de la comparaison. Tu es la chercheuse de la famille, toujours un peu ailleurs, et parfois je te l'envie cet ailleurs.

Aurore, génétiquement identique mais aussi très différente. Merci pour tes cours sur Excel et ton assistance toujours efficace. Merci d'être toi tout simplement.

Un grand merci aussi à mes oncles, tantes, cousin, cousines pour votre présence tout au long de ces années.

Au CESAP, sorte de grande famille parallèle depuis mon enfance. Mon regard sur le soin passe forcément par ce prisme, je ne serai surement pas la personne et le soignant que je suis sans vous tous, famille et soignants.

\section{A louis,}

Pas toujours facile pour un non-médecin de comprendre tout ce charabia, mais ça ne te fais 
pas peur. Merci pour ton soutien logistique au cours de la préparation de cette thèse et pour l'organisation du pot, puis pour tout le reste.

Un grand merci aussi à toute ta famille, les vosgiens et exilés que ce soit à Manosque, Brest ou ailleurs, l'accueil est toujours excellent.

\section{Aux équipes soignantes,}

J'en ai rencontré des médecins, infirmiers et Aides-soignants au cours de mes études, impossible de citer tous les noms sans en oublier. Merci donc à vous tous qui m'avez tout appris.

Des réanimations chirurgicales et médicales où je suis passées, on y rencontre de fortes personnalités tant chez les médecins que chez les infirmiers, AS, ASH. Vous m'avez donné envie de faire ce métier, d'appartenir à votre équipe. Merci pour tous vos enseignements chacun à votre niveau, petit à petit vous avez formé le soignant que je suis devenu.

Des blocs opératoires, l'équipe du PU évidemment, sans qui cette étude n'aurait pas pu avoir lieu. Ça a été facile de faire cette étude, grâce aux IADEs qui m’ont fait les mesures et gérée l'intendance (merci Maylis), et puis on y est toujours bien accueillis. Merci aussi aux équipes de tous les autres blocs opératoires qui m'ont aidé à grandir et m'ont fait aimer l'anesthésie de mon début au bloc digestif, en passant par Annecy, Chambéry, le TCCR, le cardiaque et maintenant la pédiatrie.

\section{Aux non soignants,}

Parce que l'hôpital ce n'est pas que des soignants. Un grand merci aux secrétaires du PAR et tout spécialement à Sandrine, Corinne, Stéphanie, vous m'avez rendu la tâche plus facile, en me fournissant les dossiers, en me prêtant vos bureaux pour le recueil de données, et pour toutes ces petites choses qui font que vous nous êtes indispensables.

\section{A l'équipe du CESAR,}

Il paraît que j'avais besoin de fluidifier mes explications alors j'ai décidé d'aller m'entrainer au CESAR. Merci de m'avoir permis de renouer avec la tradition familiale (je parle de l'enseignement...), c'est un plaisir de travailler sur un projet en perpétuel développement et dans la bonne humeur permanente. 


\section{Aux copains,}

Ceux rencontrés à l'hôpital, ils sont de ceux qui répondent toujours présents dans les coups durs mais aussi pour les apéros. Aux copains de l'externat, en particulier Pauline, pour nos longues heures de révision derrière un bureau et discussion au bord de l'eau. Puis à ceux de l'internat: pintades et assimilées, Améloche, lulu, cricri, zaza et la bardoune qui m’a supporté comme boulet pendant 3 mois, la RNC n'aurait pas été pareil sans toi. Aux copains de promo tibo, yvo, lucho, amélache et de dessous Jules, FF, Marion et Perrine (ma co-année recherche) parce que le boulot c'est plus sympa avec les copains. Vive l'HGN. Merci aux plus jeunes (lara, manue, béné, clemence, delphine, romain's, pier ...) de m’avoir supporté en garde, je sais bien que ce n'est pas toujours facile le concept de démocratie dictatoriale. Et bien sur un grand merci à la bande de filles d'Annecy (béa, marion + jeff, amélie, julie, auré et Caroline Dupuy), on fait de belle rencontre en périph, vous en êtes la preuve.

A la bande des loulous\&co (wash, malika, aurélie, tony (je serai devant à la transju'), clo, gilles, ana, armand, ben, superG, Renaud, Claire, PA, AM) il est bon de sortir du monde médical. Désolé mes présentations ppt n'ont rien à voir avec les vôtres et je ne gère pas toutes les subtilités d'Excel, mais vous avez encore des années pour m’apprendre tout ça. Mais au moins je me suis améliorée dans les explications pour les jeux au cours de mon internat, je sais bien que pour vous c'est bien le seul progrès que j'ai fait durant toutes ces longues études.

Aux copains anciens et récents, parisiens et grenoblois : Astou, Meuh, Adrien, Véro, Marie, jen, remi, tonio, In, pierrot, arthur, gerald, laurent parce que tout simplement c'est un plaisir à chaque fois de discuter avec vous et de passer du temps ensemble. 
Introduction et rappels bibliographiques .14

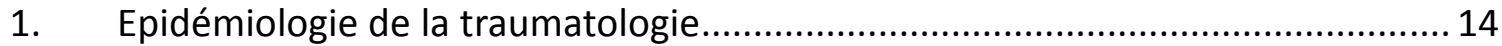

2. Outils actuels d'évaluation de la gravité d'une hémorragie :.............................. 14

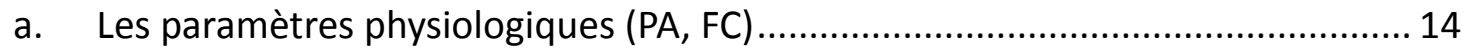

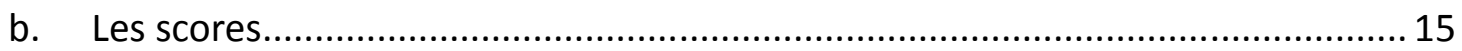

c. Mesure de l’hémoglobine capillaire : HemoCue ${ }^{\circledR}$................................................... 16

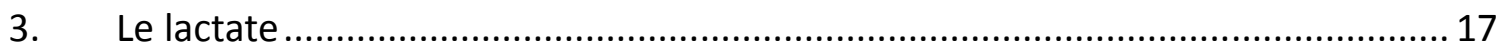

a. Formation du lactate en cas d'hypoxie cellulaire ............................................. 17

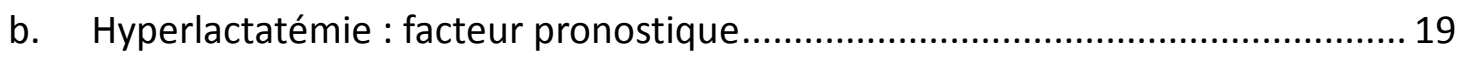

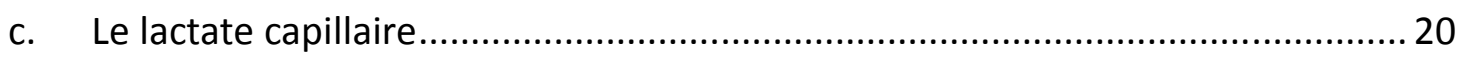

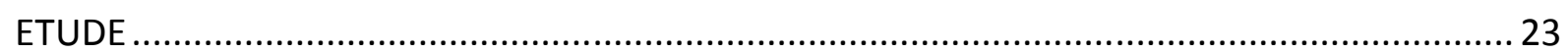

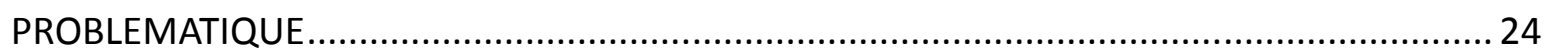

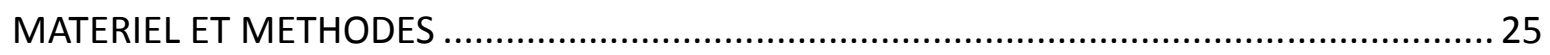



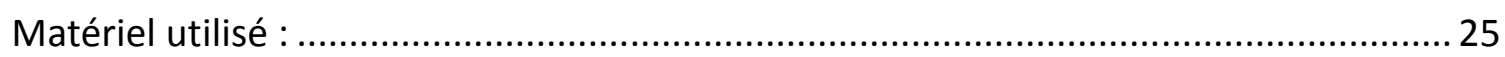

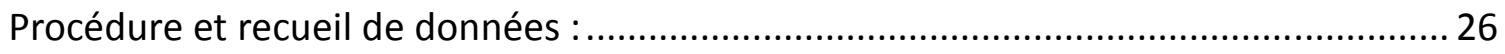

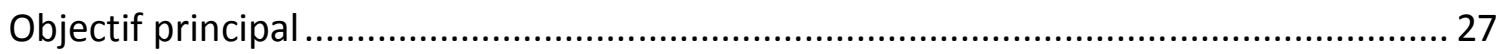

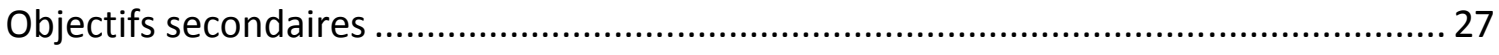

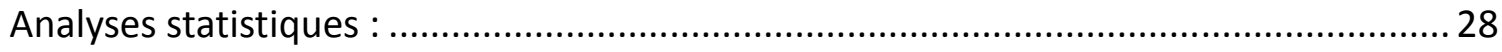

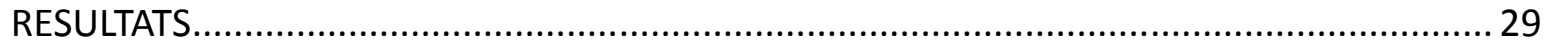

Population étudiée et analyse uni-variée des paramètres ...........................................29

Étude de l'interchangeabilité des méthodes entre le lactate capillaire et le lactate

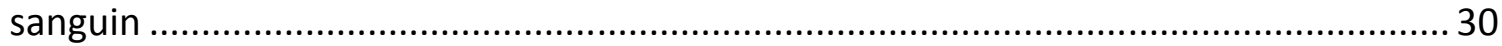

Évaluation des performances prédictives de transfusion ........................................... 31

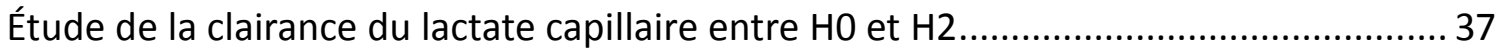

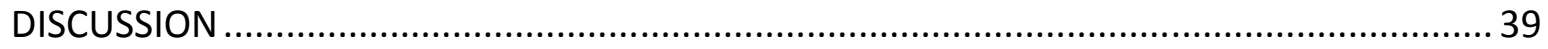

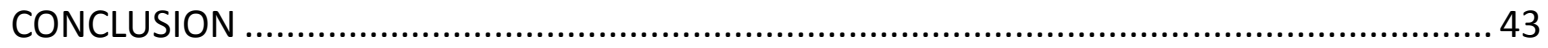

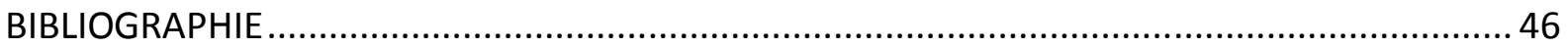

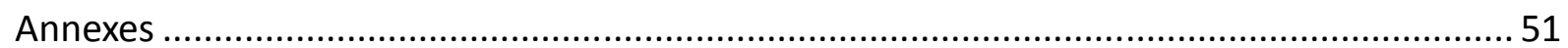


$A B C$ score: Assessment of Blood Consumption score

ASC: Aire sous la courbe

CGR: Concentré Globulaire Rouge

DCA: Déchocage

DMS: Durée Moyenne de Séjour

FC: Fréquence Cardiaque

IADE: Infirmier Anesthésiste Diplômé d'Etat

IMC: Indice de Masse Corporelle

ISS: Injury Severity Score

LDH: Lactate DésHydrogénase

MGAP: Mechanism, Glasgow, Age, arterial Pressure Score

O2: Oxygène

PAS: Pression Artérielle Systolique

PH: Pré Hospitalier

PWH score: Prince of Wales Hospital score

RTS: Revised Trauma Score

ROC: Receiver Operating Characteristic

SOFA: Sequential Organ Failure Assessment

SOFAcv: Sequential Organ Failure Assessment Cardio Vascular

SMUR: Service Mobile d'Urgence Réanimation

TRISS: Trauma Related Injury Severity Score

TASH score: Trauma Associated Severe Hemorrhage score

TDM: Tomodensitométrie 
generalites

13 


\section{Introduction et rappels bibliographiques}

\section{Epidémiologie de la traumatologie}

La traumatologie est la troisième cause de décès au niveau mondial après les maladies cardio-vasculaires et les maladies infectieuses, représentant 5,1 millions de décès par an. Son impact socio-économique est d'autant plus important qu'elle est la première cause de décès chez les moins de 40ans dans les pays développés $(1,2)$.

La majorité des décès (81\%) survient dans les 48 premières heures soit par choc hémorragique, soit par traumatisme crânien grave (3). Environ $40 \%$ des décès sont dus à une hémorragie non contrôlée. Les décès tardifs sont liés majoritairement (61\%) à des dysfonctions d'organes secondaires. Ces dysfonctions d'organes sont dues à une hypoperfusion tissulaire par chute des apports en oxygène.

La recherche et le traitement d'une hémorragie active est donc un point crucial dans la prise en charge d'un patient traumatisé pour empêcher l'apparition d'un choc hémorragique réfractaire et pour prévenir l'hypoperfusion tissulaire responsable d'une mortalité retardée importante.

Le traitement d'une hémorragie, outre l'arrêt du saignement repose sur la transfusion sanguine de Culot Globulaire Rouge (CGR). D'après le registre allemand de traumatologie, $8,1 \%$ des patients traumatisés reçoivent une transfusion massive dans les 24 premières heures (4). Près d'un quart des patients du registre reçoivent moins de 10 CGR dans les 24 premières heures et ont une mortalité précoce entre 10 et $15 \%$ et une mortalité retardée entre 20 et 30\%. Ces données soulignent l'importance d'identifier les patients qui nécessiteront une transfusion qu'elle soit ou non massive.

\section{Outils actuels d'évaluation de la gravité d'une hémorragie :}

\section{a. Les paramètres physiologiques (PA, FC)}

Lors d'une hémorragie, les mécanismes de régulation de la pression artérielle entrent en jeu très rapidement pour maintenir une circulation cérébrale, coronaire et rénale constante. La réduction du volume sanguin par l'hémorragie entraîne une réduction du retour veineux et donc devrait entraîner une baisse du débit cardiaque et de la pression 
artérielle. Néanmoins à la phase initiale il y a une stimulation des barorécepteurs carotidiens, aortiques et splanchniques induisant une réaction sympathique efférente. Au niveau périphérique il existe aussi une stimulation du système nerveux adrénergique. Ces deux mécanismes d'adaptation entraînent une vasoconstriction périphérique compensatrice permettant de normaliser la pression artérielle.

La chute de la pression artérielle est donc en générale tardive et est un mauvais reflet de la sévérité du saignement. La baisse de la pression artérielle ne survenant que pour une réduction de la masse sanguine de 30 à $50 \%$ (5).

La tachycardie est un témoin précoce de la diminution du retour veineux, mais son interprétation est difficile à la phase aigüe en raison de l'anxiété et/ou de la douleur du patient traumatisé. De ce fait la tachycardie isolée est un signe dont la sensibilité et la spécificité sont insuffisantes dans le choc hémorragique.

La difficulté de l'interprétation de ces 2 paramètres peut conduire à sous-estimer la spoliation sanguine et donc le degré d'hypoperfusion tissulaire associée. Une hypoperfusion tissulaire significative pouvant survenir avec des paramètres vitaux conservés (6).

Un Shock Index, ratio de la fréquence cardiaque sur la pression artérielle systolique, supérieur à 0,9 semble être néanmoins prédictif d'une transfusion massive chez les patients traumatisés stable hémodynamiquement (7).

\section{b. Les scores}

Pour améliorer la reconnaissance d'une hémorragie lors de la prise en charge de patients traumatisés des outils ont été développés avec l'instauration de scores de prédiction transfusionnelle. Ces scores prédictifs concernent principalement la transfusion massive (10CGR ou plus) dans les 24 premières heures. L'étude de Brockamp (8) a comparé la validation de 6 scores différents (TASH, ABC, PWH score, Vandromme score, Schreiber, Larsen score). Ces 6 scores ont été appliqués au registre allemand de traumatologie de manière rétrospective. Cette étude retrouve une bonne corrélation entre les différents scores et la transfusion de 10 CGR ou plus. Néanmoins ces scores ne sont pas applicables en pré-hospitalier car ils incluent des résultats d'examens biologiques ou d'imageries. Seul le score $A B C$ de Nunez (9) est applicable dès la prise en charge du patient à la condition d'équipes pré-hospitalières pourvues d'un appareil d'échographie. 
Ces scores permettent donc d'identifier des patients présentant une hémorragie massive nécessitant la transfusion d'au moins 10 CGR après leur admission hospitalière. Cependant ces patients les plus graves ne représentent qu'une minorité des patients victimes d'un traumatisme et la morbi-mortalité des patients nécessitant moins de 10 culots globulaires est élevée (4). En pratique clinique, le médecin pré-hospitalier et/ou au déchocage est bien plus souvent confronté à un patient traumatisé avec des paramètres physiologiques normaux ou normalisés mais dont le mécanisme lésionnel est suspect d'hémorragie. Il se pose alors le problème de l'orientation de ces patients, appelée triage. Un " sur-triage » engendre une surcharge des équipes d'urgences type déchocage et un surcoût par un ratio personnel médical/patient important. Et a contrario le " sous-triage " expose au risque de ne pas identifier un patient présentant un saignement à bas bruit qui risque de développer une hypoperfusion tissulaire, entraînant ensuite une dysfonction d'organes secondaire responsable d'une mortalité retardée.

Les scores d'aide au triage tel que le Triage Revised Trauma Score (RTS), le Trauma Related Injury Severity Score (TRISS) et le MGAP (Mécanism, Glasgow coma scale, Age, arterial Pressure) ont montré une bonne corrélation entre leur élévation et la mortalité hospitalière mais ne permettent pas de distinguer les patients victimes d'une hémorragie ou d'un traumatisme crânien grave.

\section{c. Mesure de l'hémoglobine capillaire : HemoCue}

L'HemoCue $^{\oplus}$ (AB Leo Diagnostics, Sweden) est un appareil portable qui permet la mesure de I'hémoglobine à partir de prélèvements capillaires par une méthode à l'acide de méthémoglobine et une lecture photométrique. La fiabilité du résultat et sa reproductibilité dépendent beaucoup de la technique de prélèvement. Une meilleure corrélation avec les automates de laboratoire étant retrouvée avec des prélèvements veineux ou artériels, le prélèvement capillaire pouvant manquer de fiabilité en cas de choc. (10)

L'HemoCue est un test rapide en urgence, mais il n'est pas recommandé à l'heure actuelle de l'utiliser comme critère unique de mise en place d'une transfusion. La Société Française d’Anesthésie Réanimation propose de réaliser en parallèle une numération sanguine classique.

De plus l'HemoCue, à la phase initiale de prise en charge, renseigne sur une valeur d'hémoglobine à un instant donné, et ne permet pas de déterminer l'importance de la perte 
sanguine, l'hémoglobine de référence du patient n'étant pas connue. L’hémoglobine étant une concentration, lors d'un saignement en l'absence de remplissage vasculaire le patient perdant du sang total et non seulement des globules rouges, l’hémoglobine reste stable, et donc peut être faussement rassurante.

D’autre part l'hémoglobine capillaire ne permet pas d'évaluer l'hypoperfusion tissulaire liée à l'hémorragie, et donc le retentissement de la perte sanguine. Le développement d'une souffrance puis d'une dysfonction d'organe n'apparaissant pas pour tous les patients, pour la même spoliation sanguine.

\section{Le lactate}

\section{a. Formation du lactate en cas d'hypoxie cellulaire}

La glycolyse correspond à l'ensemble des voies métaboliques énergétiques permettant la phosphorylation de l'ADP en ATP. Les étapes cytosoliques de la glycolyse conduisent à la production de pyruvate, métabolite intermédiaire essentiel. En présence d'oxygène, la majorité du pyruvate entre dans la mitochondrie pour rejoindre le cycle de Krebs et l'oxydation phosphorylante avec au final la production de 36 molécules d'ATP.

Par définition, l'hypoxie bloque l'oxydation phosphorylante mitochondriale du pyruvate de sorte que la synthèse d'ATP est inhibée. La seule issue métabolique du pyruvate est sa réduction en lactate par la lactate déshydrogénase (LDH), qui s'associe à une baisse du rapport ATP/ADP et une élévation du rapport NADH/NAD induisant une inhibition des autres voies métaboliques du pyruvate (inhibition phosphofructokinase (PFK), de la pyruvate carboxylase et de la pyruvate déshydrogénase). L'augmentation de la production de lactate en situation anaérobie résulte de l'accumulation de pyruvate qui se transforme en lactate suite aux modifications du potentiel redox. Cette conversion permet de régénérer du NAD+, permettant la production d'ATP par la glycolyse anaérobie, production qui reste cependant nettement moins efficiente sur un plan énergétique (2 ATP vs 36 ATP). II est important de considérer que la modification du potentiel redox induite par l'augmentation du rapport NADH/NAD active la transformation du pyruvate en lactate et élève par conséquent le rapport pyruvate/lactate.

$\mathrm{Au}$ total, le métabolisme énergétique anaérobie se caractérise par une hyperlactatémie à rapport pyruvate/lactate élevé, une utilisation accrue du glucose et un 
faible rendement énergétique.



Voies métaboliques : Le pyruvate peut être métabolisé dans le cytosol en se transformant en glucose par la voie de la néoglucogénèse Zou en se transformant en alanine par transamination 2 . II peut aussi être métabolisé dans la mitochondrie soit en suivant la voie oxydative du cycle de Krebs suivie

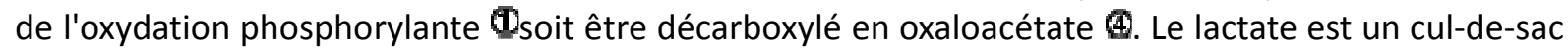
métabolique résultant d'une réaction réversible d'oxydation du pyruvate par la lacticodéshydrogénase.

PFK : phosphofructokinase; PK : pyruvate kinase; LDH : lacticodéshydrogénase.; PDH pyruvate déshydrogénase. NAD : nicotinamide adénine dinucléotide.

La lactatémie reflète l'équilibre entre production et utilisation du lactate. La production journalière normale de lactate représente 1300 à $1500 \mathrm{mmol} / \mathrm{J}$ et provient d'organes dits "producteurs " correspondant à un travail anaérobie (érythrocyte, intestin, cerveau, peau, muscles). Dans les conditions normales cette production est totalement compensée par la métabolisation hépatique. Le seuil de saturation hépatique est extrêmement élevé, la vitesse d'élimination du lactate atteint un plateau à partir d'une concentration supérieur à $5 \mathrm{mmol} / \mathrm{L}$. Hors situation de stress la production de lactate est égale à son élimination, conduisant à une lactatémie stable entre 0,5 et $1 \mathrm{mmol} / \mathrm{L}$ chez un adulte avec une demi-vie plasmatique d'environ 10 minutes. 


\section{b. Hyperlactatémie : facteur pronostique}

De nombreux travaux ont montré une relation entre sévérité de l'hyperlactatémie et la morbi-mortalité des patients de réanimation (11) et en état de choc septique $(12,13)$. L'équipe de Baker a montré chez 87 patients en choc septique que la lactatémie d'admission était de 9,6 \pm 5,3 mmol/L chez les patients décédés, alors qu'elle était plus basse $(5,6 \pm 3,7$ $\mathrm{mmol} / \mathrm{L}$ ) chez les survivants (12). La lactatémie à l'admission est un indicateur pronostique fiable mais l'étude de Baker(12) met aussi en évidence que la durée d'hyperlactatémie (temps de normalisation) est un indice de gravité tout aussi fiable et important. La durée de l'acidose lactique étant dans cette étude le meilleur paramètre prédictif de survie globale et de survenue de défaillances d'organes.

Dans les études s'intéressant à l'hyperlactatémie suite à un traumatisme, il est retrouvé au niveau expérimental (14-16), mais aussi clinique $(17,18)$, une forte relation entre la morbi-mortalité et l'importance de l'hyperlactatémie. L'étude de Moomey(14) retrouve également que contrairement aux paramètres d'oxygénation habituels (Sv02), la lactatémie est liée à la sévérité du traumatisme et à l'importance de l'hémorragie dans un modèle de choc hémorragique après traumatisme pénétrant chez le cochon.

La durée de I'hyperlactatémie évaluée chez 129 patients traumatisés est un indicateur fort de morbidité avec une hyperlactatémie prolongée chez les patients présentant une ou plusieurs défaillances d'organes (17). Une étude récente prospective retrouve qu'une clairance du lactate de moins $20 \% / h$ entre l'entrée du patient (HO) et deux heures après sa prise en charge $(\mathrm{H} 2)$, est un facteur prédictif important et indépendant de mortalité, de gravité (ISS>15) et de transfusion massive (supérieur à 6CGR en 24h) chez les patients traumatisés (18). L'analyse en sous-groupe de cette étude portant sur 462 patients normo tendus ne retrouve pas d'intérêt à la lactatémie à l'entrée et à la clairance du lactate par rapport aux scores TRISS, RTS et MGAP en termes de prédiction de la mortalité. La mortalité retrouvée chez ces patients était de 6\%, ISS moyen à 14 [8-25].

D’autres études en traumatologie se sont intéressées exclusivement aux patients traumatisés stables hémodynamiquement. L'étude rétrospective de Callaway(19) en 2009 
portant sur des patients âgés de plus de 65 ans accueillis au déchocage, stables hémodynamiquement (PAS>90 $\mathrm{mmHg}$ ), retrouve que l'élévation du lactate sanguin à l'arrivée est proportionnellement corrélée à la mortalité hospitalière. Une autre étude montre que, même chez les patients avec des paramètres physiologiques normaux, le lactate sanguin est utile pour différencier les traumatismes mineurs des traumatismes majeurs (nécessité de transfusion sanguine ou ISS > 15 ou chute de 10 point d'hématocrite). (20)

L'étude rétrospective de Vandromme(21) en 2010, portant sur 2400 patients relie une hyperlactatémie artérielle initiale à la transfusion de plus de 6CGR dans les 24 premières heures chez le patient traumatisé stable. La population de l'étude est de $75 \%$ de traumatisme fermé et de $19 \%$ de traumatisme pénétrant. Les patients inclus étaient stables hémodynamiquement, pression artérielle systolique comprise entre $90 \mathrm{mmHg}$ et $110 \mathrm{mmHg}$. Une hyperlactatémie artérielle initiale supérieure à $2,5 \mathrm{mmol} / \mathrm{I}$ multipliait par deux le risque de transfusion de 6 CGR ou plus.

\section{c. Le lactate capillaire}

La méthode de référence de mesure du lactate est celle effectuée à partir du sang artériel en laboratoire. Le résultat étant long à obtenir il a été développé des appareils déportés. Les appareils à gaz du sang permettent actuellement de mesurer la lactatémie, méthode validée par la littérature. Les résultats de ces deux techniques, en laboratoire et sur machine à gaz du sang sont obtenus respectivement en 85 minutes et en 10 minutes (22). Au cours d'une prise en charge en urgence d'un patient traumatisé, le délai d'obtention des résultats est primordial pour la prise en charge et le devenir du patient. Le développement de techniques au lit du patient répond à cet impératif. De nombreuses études ont comparé les valeurs de lactate artériel obtenues par les appareils portatifs et la méthode de référence ; celles-ci montrent une bonne corrélation entre les deux méthodes pour les patients hospitalisés en soins intensifs $(23,24)$. Une étude réalisée sur sang total à l'arrivée des patients dans un centre de traumatologie montre également une bonne corrélation entre 2 appareils portatifs et la mesure réalisée au laboratoire. (25)

Néanmoins, le recueil de sang artériel ou de sang total pose le problème de voie d'abord et de la répétition des prélèvements. Plusieurs études ont recherché si un prélèvement capillaire, au doigt ou à l'oreille, donnait des résultats différents des prélèvements sanguins. II existe deux études expérimentales contradictoires. Une étude 
dans le choc septique chez le chien (26) qui retrouve une différence significative entre le prélèvement veineux et le prélèvement capillaire. Une deuxième chez le porc en choc hémorragique (27) montrant une bonne corrélation entre les deux mesures, artérielle et capillaire $\left(R_{2}=0,98\right)$. Les études cliniques réalisées chez l'homme ont été effectuées en majorité en néonatalogie, elles montrent une très bonne corrélation entre prélèvement capillaires et artériels notamment chez le nouveau-né anémié stable $(28,29)$. Une seule étude chez l'adulte compare les résultats de lactate obtenus par ponction capillaire et ponction veineuse et retrouve un coefficient de corrélation à 0,90 . La population étudiée était celle de 25 patients atteints de sepsis accueillis aux urgences (22).

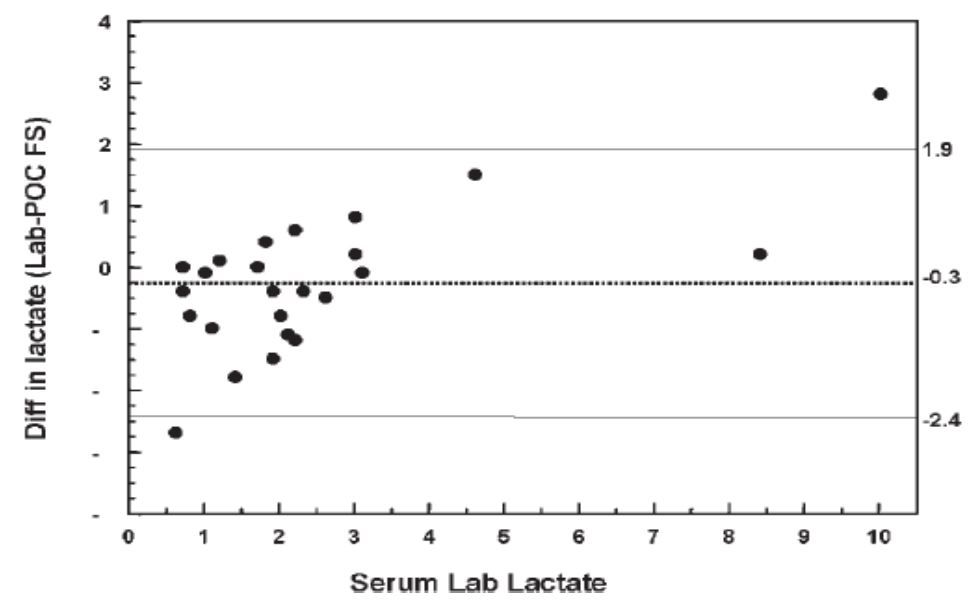

Gaeiski et al (22): Interchangeabilité de la méthode par un appareil déporté et la mesure au laboratoire de biologie. Les pointillés représentent l'intervalle de confiance à $95 \%$.

Du fait de sa facilité d'accès et de sa rapidité de résultat le lactate capillaire a été utilisé dans plusieurs études en pré-hospitalier comme facteur prédictif de gravité en traumatologie. Une corrélation très modérée a été retrouvée entre l'élévation du lactate capillaire et le score de gravité traumatologique : I'ISS (30). La majorité des études pré hospitalière mêle les résultats capillaires et veineux. Ainsi l'étude de Jansen(31) en 2008, mesure le lactate en pré-hospitalier d'origine veineuse ou capillaire. $20 \%$ des patients étudiés ont une étiologie traumatologique. L'étude retrouve une surmortalité chez les patients ayant une hyperlactatémie initiale supérieure à $3,5 \mathrm{mmol} / \mathrm{l}$ quelle que soit leur pression artérielle systolique. 


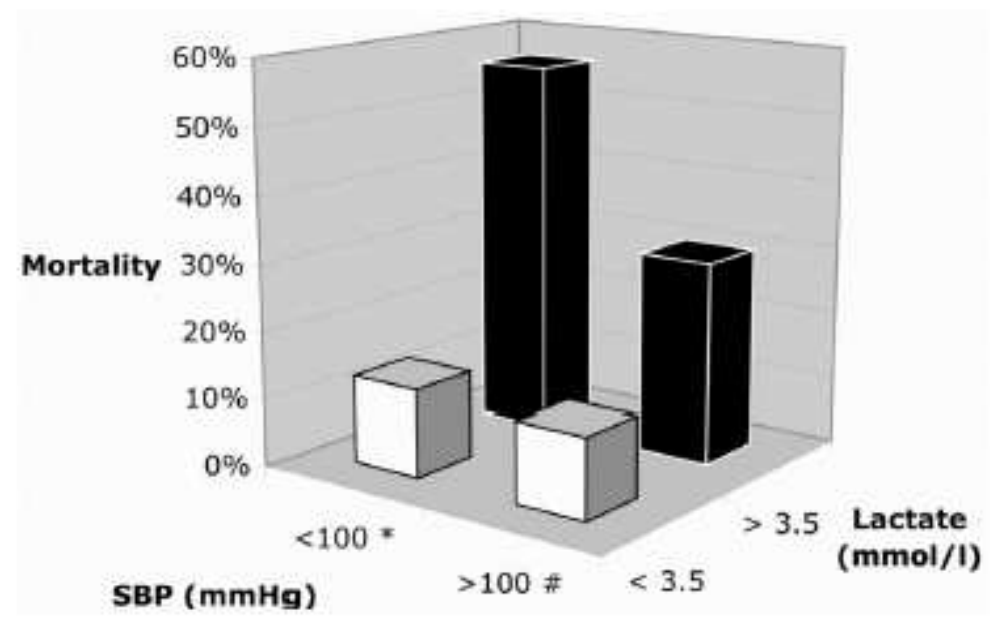

Jansen et al (31): Mortalité intra-hospitalière stratifiée selon la pression artérielle systolique (SBP : systolique Blood Pressure) et le lactate sanguin. ${ }^{*} p=0.046 \# p=0.032$. Nombre de patients par groupe : SBP basse/lactate bas $n=8, S B P$ basse/lactate élevé $n=25$, SBP élevée/lactate bas $n=58$, SBP élevée/lactate haut $n=33$.

Par ailleurs, une étude rétrospective anglaise retrouve que le lactate capillaire ou veineux à l'admission chez les patients hémodynamiquement stable est un bon facteur prédictif de transfusion de plus de 6 CGR dans les premières 24 heures et de mortalité (21). En effet dans cette étude après ajustement sur l'âge, le genre, l'ISS et le type de traumatisme, un patient avec un lactate sanguin supérieur à $2,5 \mathrm{mmol} / \mathrm{L}$ à 1,7 fois [1.1-2.7] plus de chance d'être transfusé de 6CGR par rapport à un patient ayant un lactate sanguin inférieur à 2,5mmo/l. Ce même patient a également un risque de décès 1,4 fois [0.8-2.6] plus important que les patients ayant un lactate normal (inférieur à 2,5mmol/L). Dans cette étude, plus la lactatémie s'élevait, plus le risque relatif de transfusion et de mortalité augmentait.

\begin{tabular}{|c|c|c|c|c|c|c|}
\hline \multirow[b]{2}{*}{ Variable } & \multicolumn{3}{|c|}{$\geq 6$ U PRBC in $24 \mathrm{~h}$ postinjury } & \multicolumn{3}{|c|}{ Mortality } \\
\hline & $\mathbf{n}$ & $\%$ & RR $(95 \% \mathrm{Cl})^{*}$ & $n$ & $\%$ & $\mathbf{R R}(95 \% \mathrm{Cl})^{*}$ \\
\hline \multicolumn{7}{|c|}{ Blood lactate, $\mathrm{mmol} / \mathrm{L}$} \\
\hline$\leq 2.5$ & 37 & 6.3 & - & 18 & 3.1 & - \\
\hline$>2.5-5.0$ & 54 & 15.0 & $1.7(1.1-2.7)$ & 29 & 8.1 & $1.4(0.8-2.6)$ \\
\hline$>5.0-7.5$ & 26 & 29.6 & $3.1(1.8-5.2)$ & 10 & 11.4 & $1.8(0.8-4.1)$ \\
\hline$>7.5$ & 39 & 43.8 & $4.2(2.6-6.9)$ & 27 & 30.0 & $5.1(2.7-9.7)$ \\
\hline Trend $p$ value & & & $<0.0001$ & & & $<0.0001$ \\
\hline
\end{tabular}

Vandromme et al(21): Risque Relatif ajusté du lactate sanguin pour le besoin transfusionnel de 6CGR ou plus dans les 24 heures post traumatique et la mortalité pour une PAS pré hospitalière $\geq 90$ à $110 \mathrm{mmHg}$. 
ETUDE 


\section{PROBLEMATIQUE}

La recherche d'une hémorragie, ou d'une lésion à fort potentiel hémorragique est un élément principal dans la prise en charge précoce d'un patient traumatisé grave. Lorsque le patient est instable hémodynamiquement au cours de la phase pré-hospitalière l'hémorragie est quasi certaine. La prise en charge au déchocage d'un tel patient nécessite un équipement lourd c'est-à-dire une voie veineuse centrale et une pression artérielle sanglante permettant d'optimiser l'hémodynamique du patient avant transport vers la tomodensitométrie, le bloc opératoire ou l'embolisation. Pour les patients moins graves c'est-à-dire les patients ayant été stabilisés lors de la prise en charge pré-hospitalière ou les patients stables d'emblée, la question d'une hémorragie sous-jacente avec aggravation secondaire se pose. L'intensité de prise en charge puis l'orientation sont difficiles à anticiper pour le médecin à l'accueil au déchocage. II n'existe actuellement aucun outil efficace d'aide au triage.

Une étude rétrospective (32) sur l'activité du déchocage du CHU de Grenoble entre les années 2007 à 2009, montrait que 60\% de l'activité de déchocage était d'origine traumatologique. Cela représentait pour l'année 2009, 567 patients dont 75\% étaient des patients stables ou stabilisés, soit plus de 1 patient par jour. La prise en charge de cette catégorie de patients au déchocage de Grenoble correspond donc à l'activité traumatologique principale et est une problématique quotidienne pour les médecins. Un outil objectif et rapide d'aide au triage pour ces patients serait donc utile en pratique quotidienne.

Au vu de la littérature, la mesure du lactate capillaire nous a paru intéressante dans l'aide au triage de tels patients. Celui-ci n'a jamais été évalué isolément par rapport au lactate sanguin dans la prise en charge des patients traumatisés graves hémodynamiquement stables.

Nous avons choisi d'évaluer le caractère prédictif d'un lactate capillaire supérieur à $3,5 \mathrm{mmol} / \mathrm{L}$ pour la transfusion de 4 CGR ou plus dans les 48 premières heures chez les patients stables ou stabilisés accueillis au déchocage. 


\section{MATERIEL ETMETHODES}

\section{Type d'étude et population étudiée :}

Il s'agit d'une étude prospective, observationnelle, monocentrique, réalisée au CHU de Grenoble au sein du déchocage. Elle s'est déroulée d'août 2011 à février 2013.

Après accord du Comité d'Ethique des Centres d'Investigation Clinique de l'interrégion Rhône-Alpes-Auvergne, nous avons inclus consécutivement tous les patients admis au déchocage suite à un traumatisme et dont I'hémodynamique pré-hospitalière avait été stable ou stabilisée par le SMUR (grade B ou C), PAS >90mmHg.

Les critères de non inclusion étaient un âge inférieur à 18 ans, la grossesse, un antécédent d'insuffisance hépatique sévère connue, une hypothermie $<35^{\circ} \mathrm{C}$, une transfusion pré-hospitalière, une $\mathrm{PAS}<90 \mathrm{mmHg}$ malgré un remplissage vasculaire supérieur à $1000 \mathrm{ml}$, de la noradrénaline à l'arrivée au DCA supérieur à $0,1 \mathrm{gamma} / \mathrm{kg} / \mathrm{min}$, le refus du patient.

La classification patient stable / stabilisé (grade B, grade C) utilisée pour les patients traumatisés est la même au sein du Trauma system REseau Nord Alpin des Urgences (TRENAU).

\begin{tabular}{|ll|}
\hline \multicolumn{1}{|c|}{ Stabilisé : grade B } & \multicolumn{1}{c|}{ Stable : grade C } \\
- Hypotension corrigée & - Absence d'hypotension en pré-hospitalier \\
- Traumatisme pénétrant & - Projection/ Ecrasement/ Ejection \\
- Volet thoracique & - Victime décédée ou traumatisée grave \\
- Fracture grave et hémorragique du bassin & dans l'accident \\
- Trauma vertébro-médullaire suspecté & - Accident à haute cinétique \\
- Amputation ou écrasement de membre & - Blast \\
- Brûlures > 15\% ou face ou voies aériennes & - Chute de plus de 6 mètres \\
- Traumatisme crânien glasgow < 14 & \\
\hline
\end{tabular}

\section{Matériel utilisé :}

L'appareil portable de mesure de lactate capillaire utilisé était le lactate scout $^{\circledR}$ (Senslab, Leipzig). Sa technique repose sur une mesure enzymatique et ampérométrique du lactate par la présence d'un réactif sur les bandelettes : la Lactate oxydase. Le

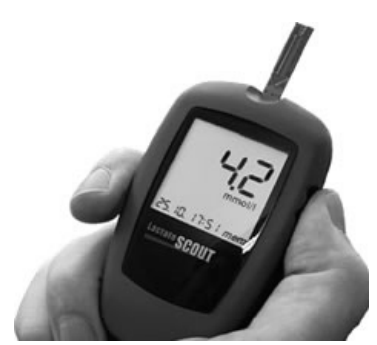


volume de sang nécessaire est de $0,5 \mu \mathrm{l}$, le temps de réalisation du test est de 10 secondes. La plage de mesure de l'appareil est de 0,8 à $20 \mathrm{mmol} / \mathrm{l}$.

\section{Procédure et recueil de données :}

L'inclusion d'un patient dans l'étude était réalisée à son entrée au déchocage, immédiatement après sa prise en charge pré-hospitalière par le SMUR. A l'arrivée du patient de façon concomitante à l'hémoglobine et à la glycémie capillaires réalisées en pratique courante, étaient effectuées deux mesures de lactate capillaire. Les mesures capillaires étaient réalisées par l'infirmier(ère) anesthésiste (IADE) en charge du patient au doigt ou au lobe de l'oreille. Après un examen rapide dans le sas du déchocage, le médecin en charge du patient répondait à un questionnaire d'appréciation clinique. Une seconde mesure de lactate capillaire était réalisée 2 heures après la prise en charge du patient.

La réalisation et le contenu du bilan sanguin fait au déchocage étaient laissés à l'appréciation du médecin en charge du patient. Ni le médecin, ni le patient n'étaient tenus au courant des résultats de la ponction. Aucune modification de traitement ou de prise en charge n'étaient décidée sur les données du lactate capillaire.

Les données suivantes étaient collectées :

- En pré-hospitalier

Age, sexe, IMC, PAS, fréquence cardiaque, volume perfusé (colloïde, cristalloïde).

- Au déchocage

Lactate capillaire, lactate veineux et/ou artériel, hémocue ${ }^{\circledR}$, hémoglobine, hématocrite, numération plaquettaire, ASAT, ALAT, TP, TCA, fibrinogène, $\mathrm{pH}$, volume perfusé colloïde et cristalloïde, transfusion CGR, utilisation d'amines vasoactives, intubation oro trachéale, pose de voie veineuse centrale, pose d'un cathéter de pression artérielle sanglante, TDM corps entier. Pression artérielle, Fréquence cardiaque, recours à une chirurgie d'hémostase ou à l'embolisation. Nombre et horaire des CGR transfusés.

- Lors de l'hospitalisation

Nombre et horaire des CGR transfusés dans les 48 premières heures, utilisation d'amines vasoactives, recours à une chirurgie d'hémostase, à l'embolisation, SOFA, SOFA cardiovasculaire. Durée de ventilation mécanique, durée d’hospitalisation en réanimation.

ISS, mortalité à J28, durée d’hospitalisation totale. 


\section{Objectif principal}

Déterminer si un lactate capillaire supérieur à 3,5mmol/L à l'arrivée au déchocage d'un patient, traumatisé grave, stable hémodynamiquement multiplie par 2 le risque d'une transfusion de 4 CGR ou plus dans les 48 premières heures.

\section{Objectifs secondaires}

- Évaluer l'interchangeabilité des mesures capillaires et sanguines lorsque réalisées

- Déterminer si l'élévation à l'arrivée du lactate capillaire est liée à la mortalité à $\mathrm{J} 28$

- Déterminer si le lactate capillaire est relié aux scores de gravité en traumatologie (ISS, SOFA)

- Comparer l'intensité de prise en charge dans les deux populations, lactate supérieur ou inférieur à 3,5mmol/L par le score d'intensité clinique

- Évaluer par une courbe ROC le seuil de lactate capillaire le plus prédictif d'une transfusion

- Comparer le lactate capillaire au score de sensibilité clinique réalisé par le médecin du déchocage

- Étudier la clairance du lactate capillaire

Le score d'intensité clinique a été établi comme suit (de 0 à 4 points) :

- Équipement veineux ou artériel central = 1 point

- Transfert en réanimation $=1$ point

- Intubation oro-trachéale au déchocage $=1$ point

- Pas d'accueil direct à la TDM = 1 point

Le score de sensibilité clinique était réalisé à l'arrivée du patient au déchocage. 3 questions étaient posées par l'IADE au médecin réanimateur en charge du patient. Score de 0 à 4.

- Pensez-vous que l'accueil direct au scanner aurait été possible? (si non +1 point) 
- Pensez-vous que le patient sera hospitalisé en réanimation au cours de sa prise en charge? (si oui + 1point)

- Pensez-vous que le patient sera transfusé ? (si oui + 1 point)

- Si oui, de plus de 4 CGR ? (si oui + 1point)

\section{Analyses statistiques :}

Le calcul du nombre de sujets nécessaires a été réalisé comme suit : sous l'hypothèse d'une sensibilité à $90 \%$, avec une prévalence de l'événement estimée à $10 \%-15 \%$ et afin d'obtenir un intervalle de confiance à $95 \%$ compris entre 83 et $95 \%$. Le nombre de sujets à inclure était de 120 patients.

La population étudiée a été divisée en 2 groupes : les patients présentant à l'arrivée un lactate capillaire supérieur ou égal à $3,5 \mathrm{mmol} / \mathrm{l}$ et ceux inférieur à $3,5 \mathrm{mmol} / \mathrm{l}$. Les critères de performance usuels (Sensibilité, Spécificité, Valeur Prédictive Positive, Valeur Prédictive Négative) ont été calculés avec un intervalle de confiance à $95 \%$.

Une analyse de type ROC a permis de déterminer si le seuil de 3,5mmol/l était le seuil optimal.

Les statistiques descriptives usuelles ont été utilisées (médiane, IQR, \%) avec utilisation du test du $\chi^{2}$ ou test $t$ de Fisher pour les variables qualitatives et un test de MannWhitney pour les variables quantitatives. Les tests statistiques ont été faits avec le risque alpha classique de $5 \%$.

La représentation graphique de Bland\&Altman a été choisie pour la comparaison des 2 méthodes : capillaire et sanguine. 


\section{Population étudiée et analyse uni-variée des paramètres}

120 patients ont été inclus sur une période de 18 mois : d'août 2011 à février 2013, au déchocage du CHU de Grenoble, après une prise en charge pré-hospitalière par les SAMU $26,38,73,74$. Les caractéristiques de cette population étudiée sont présentées dans le tableau 1. L'ISS moyen des patients inclus était de 19 (minimum 0 et maximum 51). A 48 heures, $25 \%$ des patients avaient été transfusés : $10 \%$ de 4 CGR ou plus et $15 \%$ de moins de 4 CGR.

\begin{tabular}{|c|c|}
\hline $\begin{array}{c}\text { Variables quantitative } \\
\text { ou qualitatives }\end{array}$ & $\begin{array}{c}\text { Médiane [quartile inf-sup] } \\
\text { ou } \mathrm{N}(\%)\end{array}$ \\
\hline Age (ans) & $37[27-56]$ \\
\hline Sexe masculin & $102(85 \%)$ \\
\hline IMC & $23,4[22,1-25,4]$ \\
\hline \multicolumn{2}{|l|}{ Grade } \\
\hline B & 46/119 (38\%) \\
\hline C & $73 / 119(62 \%)$ \\
\hline Temps pré-hospitalier (minutes) & $108[75-136]$ \\
\hline PAS pré-Hospitalier (mmHg) & 116 [100-131] \\
\hline FC pré-Hospitalier (/min) & 80 [71-94] \\
\hline \multicolumn{2}{|l|}{ Shock index } \\
\hline$<0,9$ & $81 / 107(75,7 \%)$ \\
\hline$>0,9$ & $26 / 107(24,3 \%)$ \\
\hline Cristalloïde pré-hospitalier (ml) & $250[0-500]$ \\
\hline Colloïde pré-hospitalier (ml) & $0[0-0]$ \\
\hline
\end{tabular}




\begin{tabular}{|c|c|}
\hline \multicolumn{2}{|l|}{ Mécanisme lésionnel } \\
\hline compression & $9(7,5 \%)$ \\
\hline décélération & $104(86,7 \%)$ \\
\hline pénétrant & $7(5,8 \%)$ \\
\hline Hémocue ${ }^{\circledR}(\mathrm{g} / \mathrm{dl})$ à l'arrivée & $13,6[12,5-14,9]$ \\
\hline Score intensité clinique & $2[1-3]$ \\
\hline Chirurgie d'hémostase DCA & $28(23,3 \%)$ \\
\hline Embolisation DCA & $4(3,3 \%)$ \\
\hline Coagulopathie à l'arrivée* & $32 / 105(65,5 \%)$ \\
\hline ISS & 19 [10-26] \\
\hline SOFA & $0[0-4]$ \\
\hline SOFA CV & $0[0-4]$ \\
\hline Transfusion $\geq 4 C G R$ à $48 \mathrm{~h}$ & $12(10 \%)$ \\
\hline Hospitalisation en réanimation & $75(62,5 \%)$ \\
\hline Infection en réanimation & $24 / 75(32 \%)$ \\
\hline Durée de séjour en réanimation (jours) & 7 [3-14] \\
\hline Mortalité à J28 & $2 / 120(1,7 \%)$ \\
\hline
\end{tabular}

Tableau 1. Caractéristiques de la population. Les variables quantitatives sont exprimées en médiane et interquartiles et les variables qualitatives en nombre de patients et en pourcentage. ${ }^{*}$ définie par Plaquette $<150$ G/L ou TP $<70 \%$ ou TCA $>1,5$.

\section{Étude de l'interchangeabilité des méthodes entre le lactate capillaire et le lactate} sanguin

L'étude de l'interchangeabilité des deux méthodes de mesure : capillaire par rapport à la mesure sanguine par la méthode de Bland et Altman, a été possible chez 60 patients à H0. On observe une variabilité importante de la mesure capillaire, avec soit une valeur 
identique soit une surestimation de la valeur. Plus les valeurs s'élèvent plus la variabilité de la mesure est importante. Le biais moyen mesuré est à $2,4 \mathrm{mmol} / \mathrm{l}$ avec une déviation standard de 2,98mmol/l. La pente de la variabilité est de 1,25. Figure 1

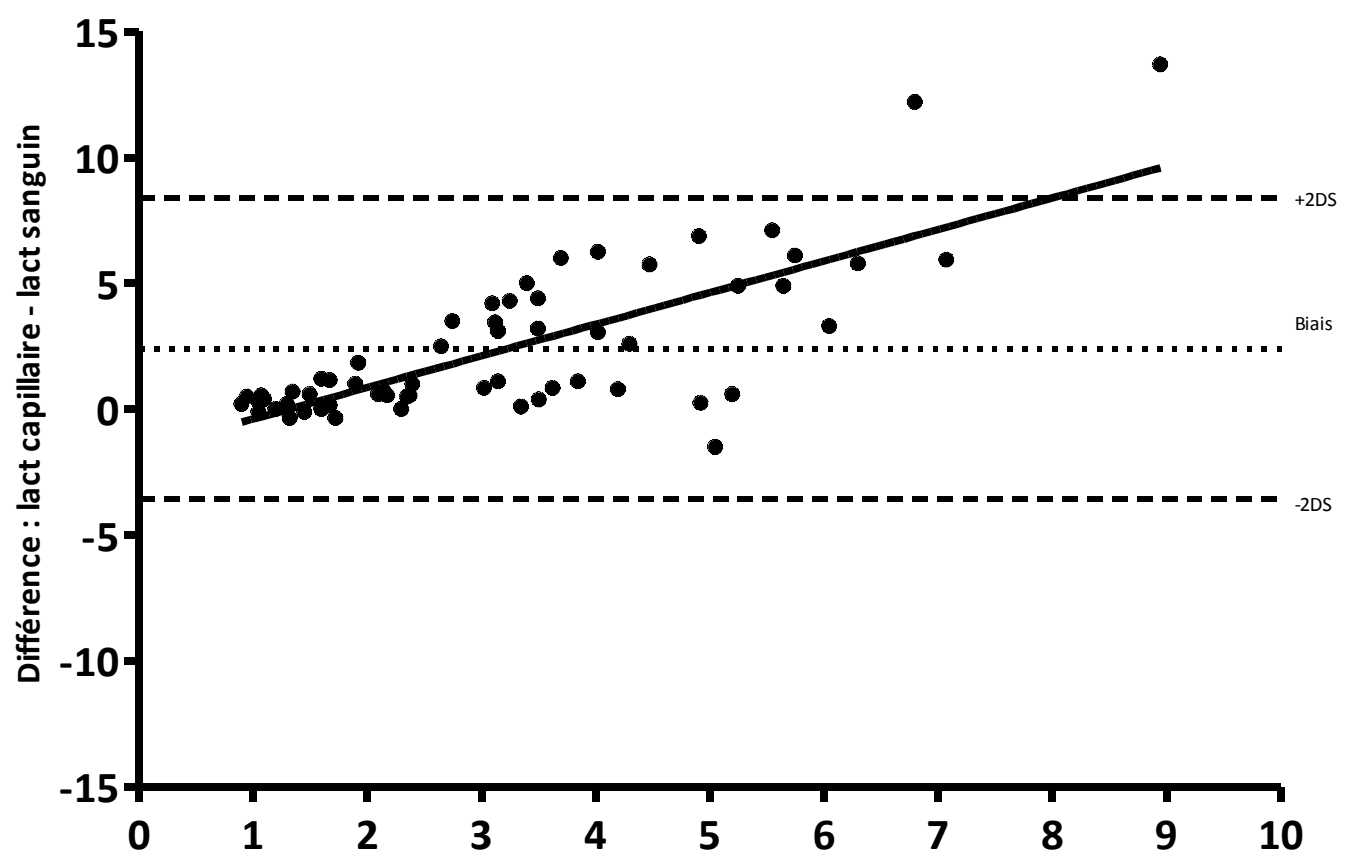

Moyenne : (lact capillaire+ lact sanguin) / 2

Figure 1 : Représentation graphique de Bland et Altman de l'interchangeabilité des deux méthodes

L'étude de la sous population, dont le lactate sanguin a été mesuré, ne montre pas de différence significative par rapport à la population n'ayant pas eu de lactate sanguin réalisé, en ce qui concerne le nombre de CGR transfusés, le score ISS, I'hémocue ${ }^{\circledR}$ à l'arrivée au déchocage, le remplissage vasculaire pré-hospitalier. En revanche une différence est retrouvée pour le score d'intensité clinique, les scores SOFA, le score de sensibilité clinique, I'hospitalisation en réanimation et la durée d'hospitalisation. (Annexe 1).

\section{Évaluation des performances prédictives de transfusion}

Deux groupes ont été constitués selon la présence ou non d'un lactate capillaire supérieur à 3,5mmol/l. Les caractéristiques démographiques et médicales des patients sont représentées dans le tableau 2.

Les paramètres significativement associés à un lactate capillaire supérieur ou égal à $3,5 \mathrm{mmol} / \mathrm{L}$ étaient la fréquence cardiaque pré-hospitalière, le mécanisme lésionnel et 
l'hémocue ${ }^{\circledR}$ à l'arrivée au déchocage.

\begin{tabular}{|c|c|c|c|}
\hline & $\begin{array}{l}\text { Lactate capillaire } \\
\qquad 33,5 \mathrm{mmol} / \mathrm{l} \\
\mathrm{N}=58\end{array}$ & $\begin{array}{l}\text { Lactate capillaire } \\
\begin{array}{c}\geq 3,5 \mathrm{mmol} / \mathrm{l} \\
\mathrm{N}=62\end{array}\end{array}$ & $p$ \\
\hline Age (ans) & $34,7[26,3-55,1]$ & $38[28,2-56,5]$ & 0,61 \\
\hline Sexe masculin & $50(86,2 \%)$ & $52(83,9 \%)$ & 0,72 \\
\hline IMC & $24,4[22,4-25,4]$ & $23,1[21,6-25,3]$ & 0,4 \\
\hline Grade & & & 0,73 \\
\hline B & $21(36,2 \%)$ & $25(40,3 \%)$ & \\
\hline C & $37(63,8 \%)$ & $37(59,7 \%)$ & \\
\hline Temps pré-hospitalier (minutes) & 90 [60-130] & $118[90-145]$ & 0,47 \\
\hline PAS pré-hospitalière $(\mathrm{mmHg})$ & $120[100-136]$ & 111 [100-129] & 0,14 \\
\hline FC pré-hospitalière (/min) & $77[69-86]$ & $82[75-100]$ & 0,02 \\
\hline Shock index (FC/PAS) & & & 0,58 \\
\hline$<0,9$ & $42(72,4 \%)$ & $42(67,7 \%)$ & \\
\hline$>0,9$ & $16(27,6 \%)$ & $2032,3 \%)$ & \\
\hline Cristalloïde pré-hospitalier (ml) & $250[0-500]$ & $250[0-500]$ & 0,26 \\
\hline Colloïde pré-hospitalier (ml) & $0[0-0]$ & $0[0-0]$ & 0,9 \\
\hline Mécanisme lésionnel & & & 0.01 \\
\hline compression & $6(10,3 \%)$ & $1(1,6 \%)$ & \\
\hline décélération & $45(77,6 \%)$ & $59(95,2 \%)$ & \\
\hline pénétrant & $7(12,1 \%)$ & $2(3,2 \%)$ & \\
\hline Hémocue $^{\circledR}(\mathrm{g} / \mathrm{dl})$ & $13,9[13,0-15,0]$ & $13,1[11,7-14,5]$ & 0,02 \\
\hline
\end{tabular}

Tableau 2. Caractéristiques démographiques et données médicales pré-hospitalières. Les variables quantitatives sont exprimées en médiane et interquartiles et les variables qualitatives en nombre de patients et en pourcentage.

Les caractéristiques d'évolution sont présentées dans le tableau 3. Une différence significative existe pour l'intensité de la prise en charge au déchocage entre les 2 groupes. Les patients présentant un lactate capillaire à l'arrivée supérieure ou égale à 3,5 mmol/L ont 
subi plus d'embolisation et présentaient plus souvent une coagulopathie. Les seuls patients qui ont été transfusés de 4 CGR ou plus étaient dans le groupe lactate capillaire supérieur ou égal à 3,5mmol/L.

II n’a pas été observé de différence en termes de mortalité (1 patient décédé dans chaque groupe), mais une durée de séjour significativement plus longue dans le groupe lactate capillaire élevé.

\begin{tabular}{|c|c|c|c|}
\hline & $\begin{array}{c}\text { Patient Lactate } \\
\text { capillaire }<3,5 \mathrm{mmol} / \mathrm{l}\end{array}$ & $\begin{array}{c}\text { Patient Lactate } \\
\text { capillaire } \geq 3,5 \mathrm{mmol} / \mathrm{l}\end{array}$ & $p$ \\
\hline Score intensité clinique & $2[1-3]$ & $2[1-4]$ & 0,04 \\
\hline Chirurgie d'hémostase DCA & $10 / 58(17,2 \%)$ & $18 / 62(29 \%)$ & 0,127 \\
\hline Embolisation DCA & $0 / 58(0 \%)$ & $4 / 62(6,5 \%)$ & 0,049 \\
\hline Coagulopathie à l'arrivée* & $10 / 50(20 \%)$ & $22 / 55(45,5 \%)$ & 0.04 \\
\hline ISS & 19 [10-26] & 18 [10-26] & 0,9 \\
\hline SOFA & $0[0-3]$ & $0[0-4]$ & 0,38 \\
\hline SOFA CV & $0[0-3]$ & $0[0-4]$ & 0,6 \\
\hline Transfusion $\geq 4 C G R$ à $48 \mathrm{~h}$ & $0 / 58(0 \%)$ & $12 / 62((19,4 \%)$ & $<0,001$ \\
\hline Hospitalisation en réanimation & $33 / 58(56,9 \%)$ & $42 / 62(67,7 \%)$ & 0,22 \\
\hline $\begin{array}{l}\text { Durée de séjour réanimation } \\
\text { (jours) }\end{array}$ & 7 [3-19] & $6,5[3,3-12,8]$ & 0,58 \\
\hline Infections & $10 / 33(30,3 \%)$ & $14 / 42(33,3 \%)$ & 0,99 \\
\hline Ventilation mécanique (jours) & $1[0-3]$ & $2[1-8,5]$ & 0,18 \\
\hline DMS (jours) & $10[5-19]$ & 17 [10-23] & 0,01 \\
\hline Mortalité J28 & $1 / 58(1,7 \%)$ & $1 / 62(1,6 \%)$ & 0,96 \\
\hline
\end{tabular}

Tableau 3. Paramètres de morbi-mortalité et scores de gravité. * définie par Plaquette<150G/L ou TP $<70 \%$ ou TCA $>1,5$

L'étude du risque relatif de transfusion chez les patients présentant un lactate supérieur à 3,5mmol/l par rapport aux patients présentant un lactate capillaire inférieur à $3,5 \mathrm{mmol} / \mathrm{l}$ est détaillé dans le tableau 4. Aucun calcul de risque relatif n'est possible pour une transfusion 4 CGR ou plus dans la mesure où il n'a pas été observé de transfusion de plus de 3 CGR dans le groupe lactate inférieur à 3,5mmol/l. 12 patients ont eu une 
transfusion de 4CGR ou plus, représentant $10 \%$ de l'effectif total, tous avaient un lactate capillaire supérieur à 3,5mmol/l.

\begin{tabular}{|cccc|}
\hline & \multicolumn{3}{c|}{ Nombre de patients } \\
Nb CGR transfusés & $\begin{array}{c}\text { Lactate capillaire } \\
<3,5 \mathrm{mmol} / \mathrm{l}\end{array}$ & $\begin{array}{c}\text { Lactate capillaire } \\
\geq 3,5 \mathrm{mmol} / \mathrm{I}\end{array}$ & RR [IC95\%] \\
$\geq 1$ & 8 & 22 & $3,43[1,38-8,53]$ \\
$\geq 2$ & 6 & 21 & $4,43[1,64-12]$ \\
$\geq 3$ & 2 & 15 & $8,9[1,94-41,08]$ \\
$\geq 4$ & 0 & 12 & \\
\hline
\end{tabular}

Tableau 4. Calcul du risque relatif de transfusion de CGR entre les 2 groupes de lactate capillaire

On observe une différence significative pour le nombre de CGR transfusés entre les deux groupes. La figure 2 représente la distribution du nombre de CGR par patient en fonction des 2 groupes.

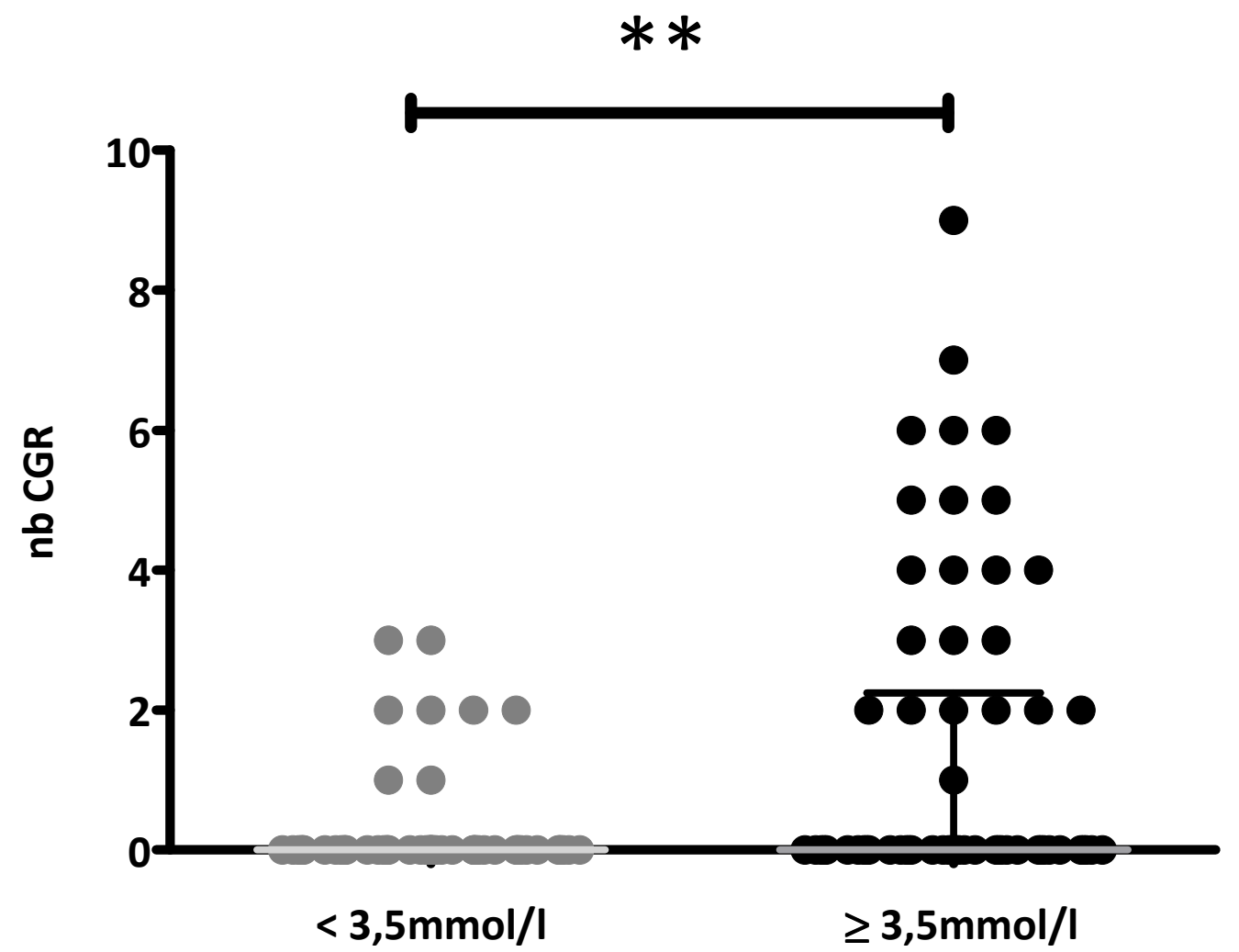

Figure 2 : Nombre de CGR transfusés dans le groupe lactate capillaire $<3,5 \mathrm{mmol} / \mathrm{l}$ et le groupe $\geq 3,5 \mathrm{mmol} / \mathrm{l}$. Chaque point représente un patient. ${ }^{* *} p<0,01$, médiane et interquartiles. 
Lors de l'étude en sous-catégorie de lactate capillaire on remarque que les patients qui ont reçu le plus de CGR sont les patients dans les catégories de lactate capillaire élevé (Figure 3).

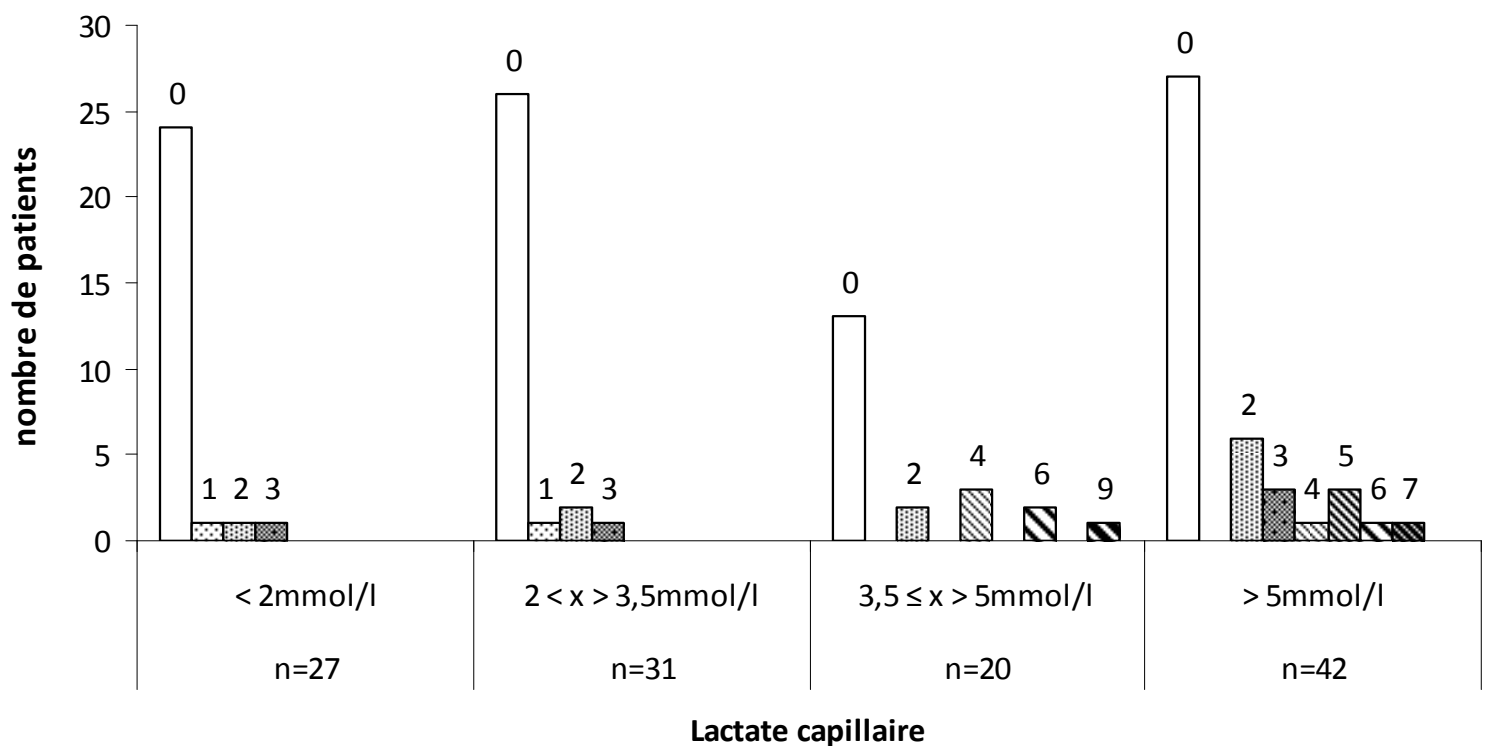

Figure 3 : Nombre de patients transfusés avec le nombre de CGR à la $48^{\text {ème }}$ heure en fonction du lactate capillaire.

Des courbes ROC ont été établies (figure 4) incluant le lactate capillaire et les autres outils de prédiction transfusionnelle (Hémocue $^{\circledR}$, Shock Index, PAS, FC) et le score de sensibilité clinique.

Les valeurs d'aire sous la courbe (ASC) sont présentées dans le tableau 5.Le lactate capillaire a une valeur prédictive équivalente au Shock Index pour la transfusion sanguine. L'élément le plus prédictif étant le score de sensibilité clinique. II n'est pas retrouvé de critère prédictif transfusionnel pour la pression artérielle systolique avec une aire sous la courbe proche de 0,5 .

A partir de la courbe ROC, la valeur seuil du lactate capillaire permettant la meilleure capacité prédictive de transfusion de 4 CGR ou plus en 48 heures était de 3,5mmol/l. Pour cette valeur, la sensibilité était de $100 \%\left(73,5 \%-100 \%\right.$ IC $\left.{ }^{95 \%}\right)$ et la spécificité de $52,8 \%$ $\left(42,9 \%-62,4 \%\right.$ IC $\left.{ }^{95 \%}\right)$. La valeur prédictive négative (VPN) retrouvée pour un lactate capillaire à 3,5mmol/L était de $100 \%$ et la valeur prédictive positive (VPP) de $19,35 \%$. 


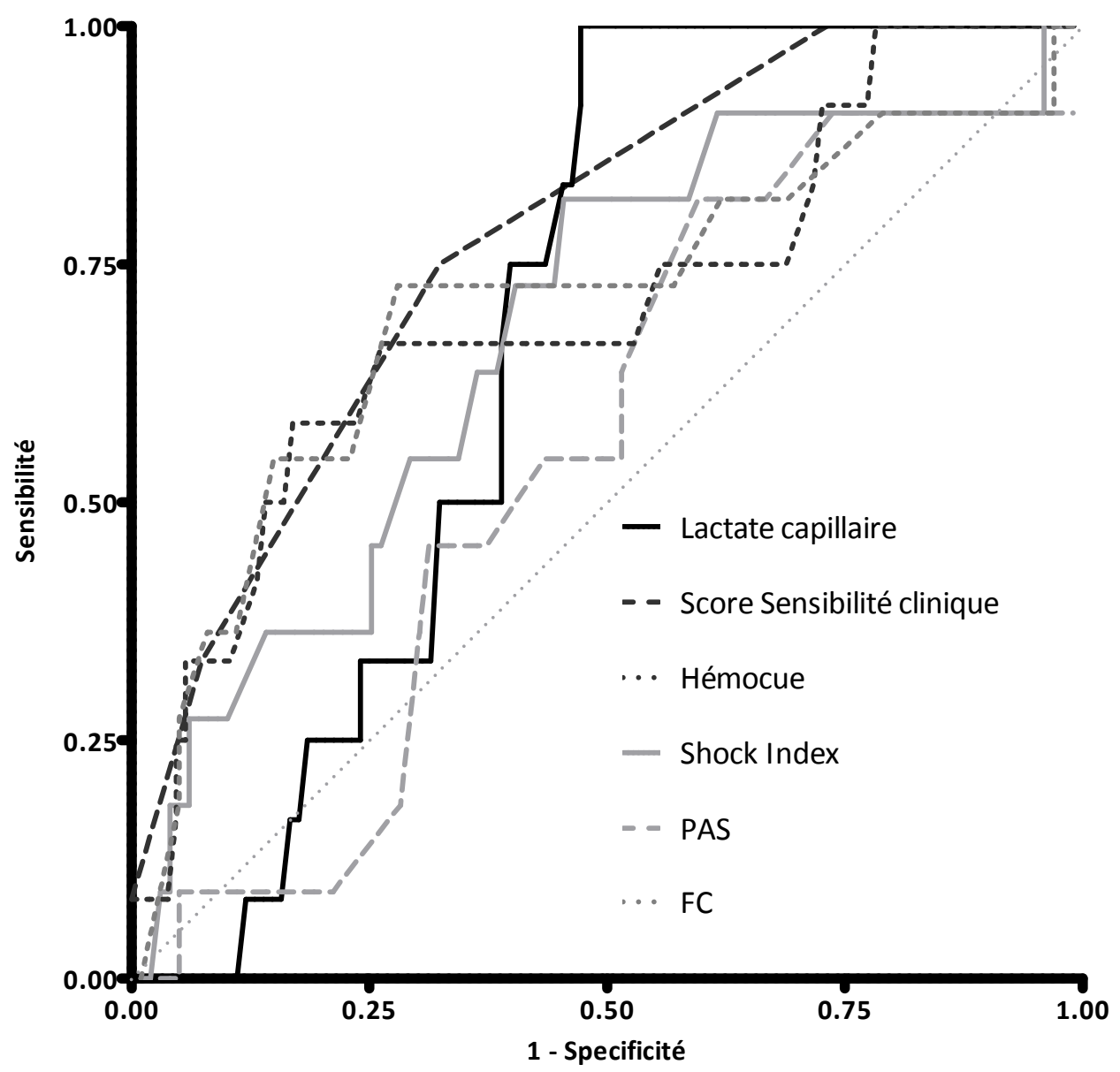

Figure 4 : Courbes ROC des principaux outils de prédiction transfusionnelle de 4 CGR ou plus

\begin{tabular}{|lcc|}
\hline & ASC & IC à 95\% \\
Lactate capillaire & 0,676 & $0,575-0,776$ \\
$\begin{array}{l}\text { Score de sensibilité } \\
\text { clinique }\end{array}$ & 0,776 & $0,650-0,902$ \\
FC & 0,705 & $0,516-0,893$ \\
Hémocue $^{\circledR}$ & 0,703 & $0,534-0,872$ \\
Shock Index & 0,679 & $0,511-0,847$ \\
PAS & 0,553 & $0,393-0,713$ \\
\hline
\end{tabular}

Tableau 5 : Valeurs des aires sous la courbe ROC pour chacun des paramètres dans la prédiction de la transfusion de 4CGR ou plus

Nous avons ensuite étudié l'intérêt de créer un score composite alliant la sensibilité clinique du médecin et le lactate capillaire. Le score composite est tel que : on ajoute un point au score de sensibilité clinique si le lactate capillaire est supérieur ou égal à 
3,5mmol/L. La courbe ROC obtenue pour la transfusion de 4CGR ou plus est représentée figure 5, l'ASC du score composite est de 0,854 (0,76-0,94 IC $\left.{ }^{95 \%}\right)$

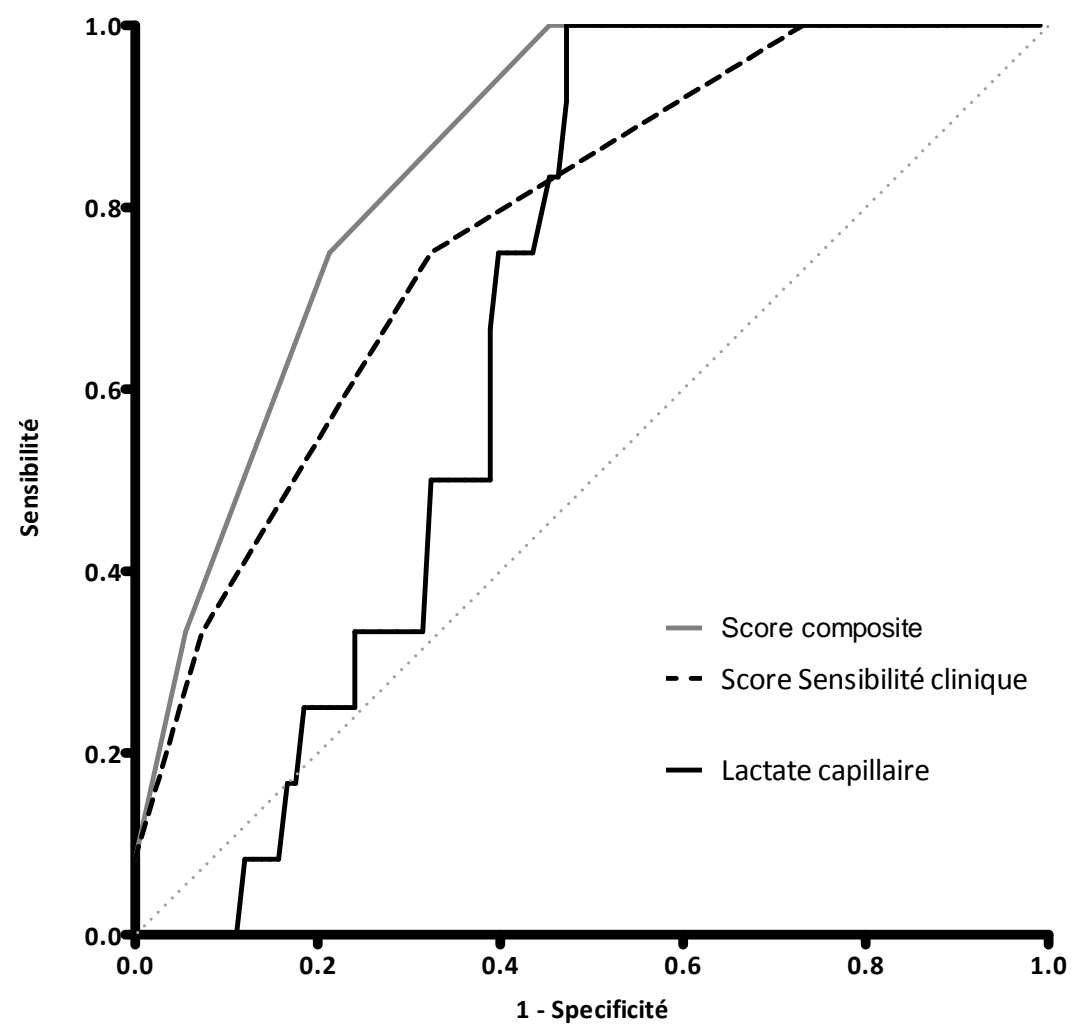

Figure 5 : Courbe ROC de prédiction transfusionnelle de 4CGR ou plus pour le score composite

L'étude des rapports pour le score composite des sensibilités et spécificités est présentée dans le tableau 6

\begin{tabular}{|lllll|}
\hline Seuil & Sensibilité $\left(\mathbf{I C}^{95 \%}\right)$ & Spécificité $\left(\mathbf{I C}^{95 \%}\right)$ & VPP & VPN \\
\hline 1 & $100(73,5-100)$ & $12(6,6-19,7)$ & $11 \%$ & $100 \%$ \\
2 & $100(73,5-100)$ & $54,6(44,7-64,2)$ & $20 \%$ & $100 \%$ \\
3 & $75(42,8-94,5)$ & $78,7(69,7-86)$ & $28 \%$ & $97 \%$ \\
4 & $33,3(9,9-65,1)$ & $94,4(88,3-97,9)$ & $40 \%$ & $93 \%$ \\
5 & $8,3(0,21-38,5)$ & $100(96,6-100)$ & $100 \%$ & $91 \%$ \\
\hline
\end{tabular}

Tableau 6 : Spécificité, sensibilité, VPN, VPP pour les différentes valeurs du score composite

\section{Étude de la clairance du lactate capillaire entre $\mathrm{HO}$ et $\mathrm{H} 2$}

Une mesure de lactate capillaire à $\mathrm{HO}$ et à $\mathrm{H} 2$ a été réalisée chez 94 patients. 4 patients ont été transfusés entre $\mathrm{HO}$ et $\mathrm{H} 2$, ils ont donc été exclus de l'étude de la clairance. Nous avons défini un premier groupe, appelé décroissance $>50 \%$, comprenant les patients dont le lactate capillaire à $\mathrm{HO}$ était inférieur à $3,5 \mathrm{mmol} / \mathrm{L}$ et le restait à $\mathrm{H} 2$ et les patients 
dont la clairance du lactate capillaire était supérieure à $50 \%$ entre la valeur à $\mathrm{HO}$ et la valeur $\mathrm{H} 2$ et dont le lactate capillaire à $\mathrm{HO}$ était supérieur ou égal à $3,5 \mathrm{mmol} / \mathrm{L}$. Le deuxième groupe était celui des patients où il n'était pas observé de clairance du lactate (stabilité ou augmentation), ou une clairance d'une valeur inférieure à $50 \%$ en deux heures. Les résultats sont présentés à la figure 6 .

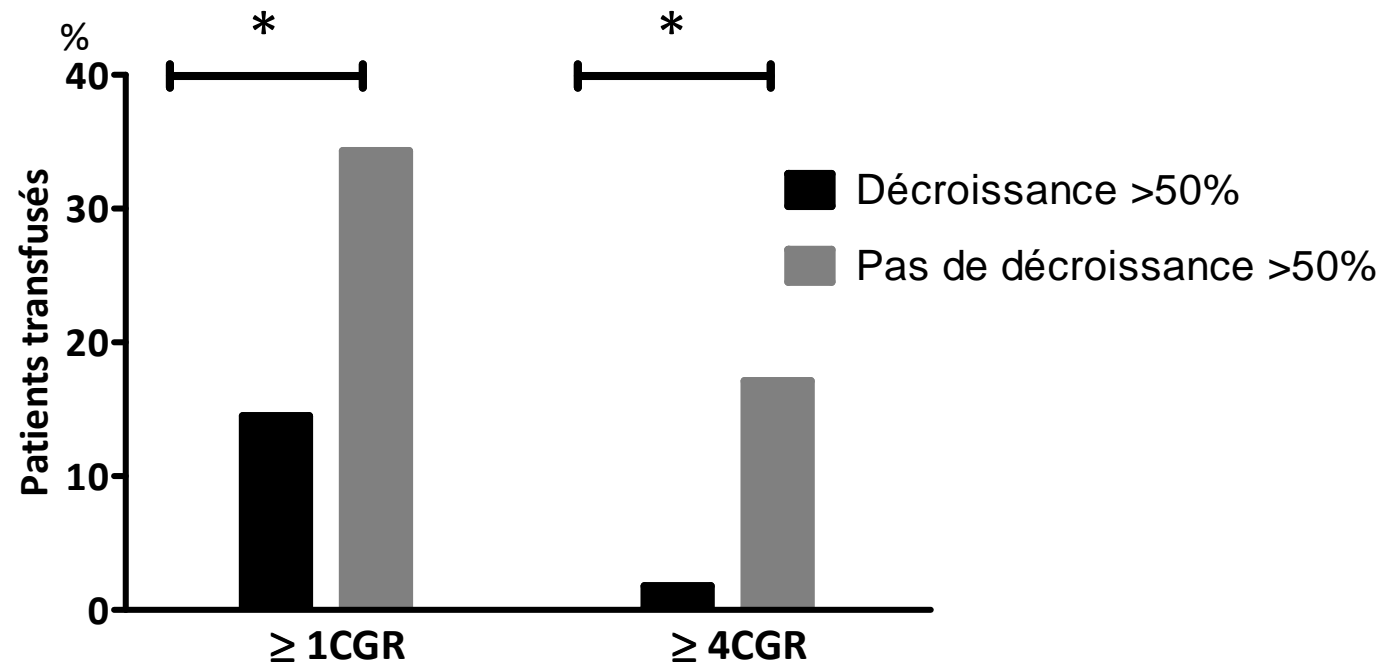

Figure 6 : Clairance du lactate. Nombre de patients transfusés de 1CGR ou plus et de 4 CGR ou plus en fonction de leur clairance du lactate ${ }^{*}: p<0,05$

Les patients du groupe décroissance $>50 \%$ présentaient significativement moins de transfusion sanguine par rapport au groupe absence de clairance de $50 \%$ du lactate capillaire que ce soit pour une transfusion de 1 CGR ou plus $(14,5 \%$ vs $34,3 \%)$, ou de 4 CGR ou plus $(1,8 \%$ vs $17,1 \%)$. 


\section{DISCUSSION}

Cette étude est la première à s'intéresser au lactate capillaire comme facteur pronostique transfusionnel chez les patients traumatisés hémodynamiquement stables. Le but de cette étude était de rechercher si une mesure de lactate capillaire supérieure à $3,5 \mathrm{mmol} / \mathrm{L}$ multipliait par 2 le risque de transfusion de 4 CGR ou plus chez un patient traumatisé grave stable hémodynamiquement accueilli au déchocage.

La population étudiée est bien celle de patients traumatisés grave, l'ISS moyen étant à 19. II existe néanmoins une forte variabilité entre les individus, l'ISS minimal étant de 0 et le maximal de 51, confirmant bien que la stabilité hémodynamique des patients peut parfois masquer des lésions importantes.

Dans notre étude une mauvaise corrélation est retrouvée entre le lactate capillaire et le lactate sanguin. La variabilité de la mesure capillaire est très importante et s'élève en même temps que la valeur moyenne de lactate. Une première explication peut venir du fait que notre étude n'avait pas pour objectif principal l'étude de l'interchangeabilité des 2 méthodes. Ainsi, les 2 prélèvements n'étaient pas réalisés de manière simultanée et systématique. Le lactate capillaire était réalisé dès l'arrivée au déchocage, le lactate sanguin nécessitant un abord veineux, pouvait être réalisé en pratique plusieurs minutes voir dizaines de minutes après. Néanmoins cette différence de temporalité ne permet pas d'expliquer des différences de valeur pouvant parfois atteindre $10 \mathrm{mmol} / \mathrm{l}$. Une explication possible à ces valeurs extrêmes est la persistance de sueur au niveau du site de ponction capillaire. En effet la ponction capillaire était réalisée dans le contexte de l'urgence à l'arrivée du patient au déchocage, le site de ponction étant parfois difficilement atteignable du fait du matelas coquille ou d'une minerve, la qualité de la ponction pouvait ne pas être optimale. Il pouvait persister de la sueur surtout dans notre population traumatisées qui correspond pour moitié à des accidents liés au sport ou à un travail physique. L'étude biochimique de la composition de la sueur montre la présence systématique de lactate et une variation importante de la concentration en lactate en fonction de l'activité physique $(33,34)$. Pour essayer de s'affranchir de ce biais, deux mesures de lactate capillaire étaient réalisées à $\mathrm{HO}$ avec pour certains patients une variabilité importante entre les 2 mesures. Nous avons effectué pour tous les patients une moyenne des 2 mesures. Cependant dans le cas de patient présentant une différence importante entre les 2 mesures, un nouveau 
nettoyage de la peau et la réalisation de nouvelles mesures auraient peut être diminué la variabilité de la mesure.

Un défaut de fiabilité de la ponction capillaire a été rapporté en cas d'œdème important ou de remplissage vasculaire massif(26), néanmoins cela ne semble pas être le cas dans notre population où le remplissage vasculaire ne dépassait pas 1 litre.

Concernant l'objectif principal de notre étude, c'est-à-dire la prédiction transfusionnelle du lactate capillaire, nos résultats montrent qu'un lactate capillaire supérieur ou égal à 3,5mmol/l est significativement associé à une transfusion plus fréquente de 4CGR ou plus. Le calcul du risque relatif de transfusion de plus de 4CGR entre le groupe lactate capillaire supérieur ou égal à $3,5 \mathrm{mmol} / \mathrm{L}$ et le groupe lactate capillaire inférieur à $3,5 \mathrm{mmol} / \mathrm{L}$ manque de pertinence au vu du petit nombre de patients transfusés de 4CGR ou plus. Néanmoins dans notre étude tous les patients ayant reçu 4CGR ou plus avaient un lactate capillaire supérieur ou égal à 3,5mmol/L. De même tous les patients ayant nécessité une embolisation au déchocage avait un lactate capillaire supérieur ou égal à 3,5mmol/L. Ces différences entre les deux groupes étaient statistiquement significatives.

Le meilleur seuil prédictif de lactate capillaire retrouvé est à $3,5 \mathrm{mmol} / \mathrm{L}$ après étude de la courbe ROC avec une spécificité à $53 \%$ et une sensibilité à $100 \%$, cela a confirmé la pertinence de notre choix pour ce seuil. La valeur prédictive positive pour ce seuil était de $19,35 \%$ et la valeur prédictive négative de $100 \%$. Cette forte valeur prédictive négative est un résultat intéressant en pratique quotidienne, elle fait du lactate capillaire un outil de triage pertinent pour le praticien. Ce résultat confirme ainsi les résultats de l'étude de Jansen(31) retrouvant une surmortalité dans le groupe lactate veineux ou capillaire supérieur à 3,5mmol/L quel que soit le niveau de pression artérielle systolique. Une autre étude s'intéressant au seuil de lactate sanguin pour la prédiction de la morbidité des patients normotendus proposait un seuil similaire à $4 \mathrm{mmol} / \mathrm{l}$ (35).

La performance diagnostique évaluée à partir de courbes ROC n'était pas optimale avec une Aire Sous la Courbe (ASC) à 0,676 (0.575 -0.776, IC 95\%). Cependant elle restait comparable à celle du Shock Index, qui est un facteur reconnu de prédiction de transfusion sanguine, ASC à 0,679 (0,511-0,847, IC95\%). La fréquence cardiaque a une ASC intéressante à 0,704 mais son intervalle de confiance est très étendu $(0,516-0,893)$ reflétant bien la difficulté d'interprétation d'une fréquence cardiaque élevée en pré-hospitalier. Le meilleur facteur prédictif retrouvé est le score de sensibilité clinique réalisé à l'arrivée du patient par 
le médecin réanimateur en charge du patient, ASC à 0,776 (0,650-0,902, IC95\%). Cela confirme la nécessité de médecins expérimentés en traumatologie grave pour la prise en charge de ces patients. Cependant le score de sensibilité clinique reste subjectif et ne ciblait pas exclusivement le risque de transfusion sanguine. Le but de notre étude était de trouver un outil objectif d'aide à l'évaluation de la gravité du patient traumatisé stable hémodynamiquement. Le score composite prenant en compte le score de sensibilité clinique et le lactate capillaire pour la prédiction transfusionnelle de 4CGR retrouve une meilleure ASC par rapport aux valeurs isolées des deux paramètres avec une ASC à 0,854 $(0,768-0,939)$. Un score composite à 3 ou plus semble être le plus pertinent avec pour ce seuil une sensibilité de $75 \%$ et une spécificité de $78,7 \%$. Lorsque l'on s'intéresse aux valeurs prédictives positives et négatives on retrouve respectivement $28 \%$ et $97 \%$, le nombre de faux positif est donc sensiblement diminué par rapport à l'utilisation du lactate capillaire seul. Par ailleurs notre étude confirme l'absence de caractère prédictif de la Pression Artérielle Systolique pour notre catégorie de patients probablement du fait des mécanismes de compensation physiologique.

Le seuil choisi de transfusion de 4 CGR est critiquable. Les études sur la transfusion chez les patients traumatisés s'intéressent principalement à la transfusion massive. Dans la littérature, celle-ci est définie comme une transfusion de 10CGR dans les 24 ou 48 ères heures. C'est donc un événement rare surtout chez les patients traumatisés stables hémodynamiquement, dans notre étude aucun patient n'a été transfusé de 10CGR ou plus. Néanmoins, des études ont montré qu'une augmentation de la morbi-mortalité survient pour des volumes transfusés beaucoup moins importants. En effet une mortalité de $20 \%$ était retrouvée chez les patients ayant reçu de 1 à 5 CGR contre 10\% pour les patients non transfusés dans l'étude de Dutton(4). Dans une étude sur le caractère prédictif du doppler splénique(36) sur la transfusion des patients traumatisés stables, le seuil transfusionnel retenu était de 2 CGR. Ce seuil de 2CGR nous a semblé trop dépendant du médecin réanimateur prenant en charge le patient et peu significatif en pratique médicale courante. Nous avons donc choisi un seuil qui nous a semblé pertinent cliniquement mais atteignable en terme de nombre de patients à inclure.

Nous avons retrouvé un caractère prédictif transfusionnel à l'absence de clairance de 
$50 \%$ du lactate capillaire sur 2 heures, résultat que l'on peut rapprocher de l'étude publiée récemment par l'équipe de Régnier (18). Cette étude s'intéressait au lactate sanguin chez tous les patients traumatisés admis au déchocage et a montré que l'absence de clairance du lactate de $20 \% / h$ était un facteur prédictif de mortalité, ceci même dans le sous-groupe des patients traumatisés normotendus. L'incidence de décès dans ce groupe de patient était de $6 \%$, et I'ISS était à 14 . Lorsque l'on applique le seuil de clairance à $20 \% / h$ à notre cohorte on retrouve 11 patients transfusés pour le groupe clairance du lactate capillaire inférieure à $20 \% / h(n=27)$, et 9 transfusions dans le groupe diminution supérieure à $20 \% / h(n=63)$, cette différence observée est statistiquement significative $(p<0,05)$. De même une différence significative est retrouvée pour la transfusion de 4CGR ou plus (1 patient vs 6 patients $p<0,05)$. La clairance du lactate est donc bien un élément pronostique important en traumatologie, mais nécessite un recul de plusieurs heures. Son utilisation en pratique clinique quotidienne reste à définir. 
CONCLUSION 
Thèse soutenue par : Clotilde Schilte

Titre : Prédiction du besoin transfusionnel chez le patient traumatisé hémodynamiquement stable par la mesure du lactate capillaire au déchocage

\section{Conclusion}

La recherche et le traitement d'une hémorragie sont la priorité du médecin prenant en charge un patient traumatisé grave. De nombreux scores existent pour identifier les patients à risque de transfusion massive, mais cela concerne peu de patients. La majorité des patients ayant subi un traumatisme grave ne va être transfusée que de quelques Culots Globulaires Rouge (CGR), néanmoins la morbi-mortalité de ces patients est plus élevée que celle des patients non transfusés. Il paraît donc important d'identifier les patients à risque de saignement et donc de transfusion. Les paramètres physiologiques tel que la pression artérielle et la fréquence cardiaque sont de mauvaix indicateurs car ils sont conservés jusqu'à une perte sanguine de $30 \%$.

Le lactate sanguin est un marqueur reconnu de morbi-mortalité et de transfusion sanguine chez les patients traumatisés. Néanmoins il nécessite pour sa réalisation un abord veineux et les résultats sont obtenus en plusieurs minutes. A l'instar de l'hémocue ${ }^{\circledR}$ pour la mesure de l'hémoglobine capillaire, la mesure du lactate par ponction capillaire s'est développée.

L'objectif de notre étude était d'évaluer si un lactate capillaire supérieur à 3,5mmol// multipliait par 2 le risque de transfusion de 4CGR ou plus chez les patients traumatisés grave stable hémodynamiquement.

II s'agit d'une étude prospective observationnelle réalisée entre août 2011 et janvier 2013 au déchocage du CHU de Grenoble où nous avons inclus 120 patients admis via le SMUR pour traumatisme grave avec une hémodynamique conservée. Dès leur arrivée au déchocage était réalisée une mesure capillaire du lactate. La prise en charge et la surveillance des patients étaient conforme aux pratiques habituelles de l'unité du déchocage

Nous avons recueilli les données de prise en charge pré-hospitalière, au déchocage et d'hospitalisation jusqu'à 28 jours après leur admission.

Nous avons obtenu 62 patients dans le groupe lactate capillaire à l'admission supérieur ou égal à $3,5 \mathrm{mmol} / \mathrm{l}$ et 58 patients dans le groupe lactate capillaire inférieur à 
3,5 mmol/I. Dans le groupe de patients "hyperlactatémie capillaire" la transfusion sanguine de plus de 4CGR était statistiquement plus importante que dans le groupe "normolactatémie capillaire". Les patients "hyperlactatémie capillaire" avaient eu plus d'embolisation, plus de chirurgie d'hémostase que les patients "normolactatémie capillaire"

Les courbes ROC établies pour définir les performances pronostiques du lactate capillaire pour la transfusion sanguine, montrent que le lactate capillaire a une Aire Sous la Courbe à $0,676\left(0,575-0,776\right.$ IC $\left.{ }^{95 \%}\right)$ meilleure que celle de la pression artérielle à 0,553 $\left(0,393-0,713\right.$ IC $\left.{ }^{95 \%}\right)$, équivalente à celle du shock index à 0,679 $\left(0,511-0,847\right.$ IC $\left.{ }^{95 \%}\right)$ et de I'hémocue ${ }^{\circledR}$ à $0,703\left(0,534-0,872 \mathrm{IC}{ }^{95 \%}\right)$ et inférieure à la sensibilité clinique du praticien en charge du patient à $0,776\left(0,650-0,902\right.$ IC $\left.^{95 \%}\right)$.

La sensibilité et la spécificité de notre valeur seuil de lactate capillaire à 3,5mmol/l pour la transfusion de 4CGR à 48 heures étaient respectivement de $100 \%$ et de $52,8 \%$. La valeur prédicitve négative pour la transufusion de 4CGR était de $100 \%$ pour ce seuil à $3,5 \mathrm{mmol} / \mathrm{L}$.

Nous avons également évalué l'interchangeabilité des deux méthodes capillaire et sanguine pour 60 patients. Le biais moyen retrouvé est à 2,4mmol/l.

En conclusion, un lactate capillaire inférieur à $3,5 \mathrm{mmol} / \mathrm{L}$ a une très bonne valeur prédictive négative concernant la transfusion de 4CGR ou plus. Le lactate capillaire peut donc être un outil intéressant dans l'évaluation des patients traumatisés graves stables hémodynamiquement.

Vu et permis d'imprimer

Grenoble, le 18 k112013

Le Doyen

M. le Professeur J.P. Romanet

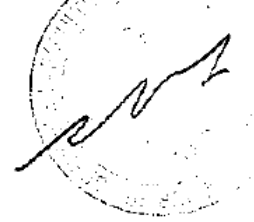

Le président de thèse

M. le professeur J.F. Payen




1. Krug EG, Sharma GK, Lozano R. The global burden of injuries. Am J Public Health. avr 2000;90(4):523-526.

2. Murray CJ, Lopez AD. Mortality by cause for eight regions of the world: Global Burden of Disease Study. Lancet. 3 mai 1997;349(9061):1269-1276.

3. Sauaia A, Moore FA, Moore EE, Moser KS, Brennan R, Read RA, et al. Epidemiology of trauma deaths: a reassessment. J Trauma. févr 1995;38(2):185-193.

4. Dutton RP, Lefering R, Lynn M. Database predictors of transfusion and mortality. J Trauma. juin 2006;60(6 Suppl):S70-77.

5. Schadt JC, Ludbrook J. Hemodynamic and neurohumoral responses to acute hypovolemia in conscious mammals. Am J Physiol. févr 1991;260(2 Pt 2):H305-318.

6. Scalea TM, Holman M, Fuortes M, Baron BJ, Phillips TF, Goldstein AS, et al. Central venous blood oxygen saturation: an early, accurate measurement of volume during hemorrhage. J Trauma. juin 1988;28(6):725-732.

7. Vandromme MJ, Griffin RL, Kerby JD, McGwin G Jr, Rue LW 3rd, Weinberg JA. Identifying risk for massive transfusion in the relatively normotensive patient: utility of the prehospital shock index. J Trauma. févr 2011;70(2):384-388; discussion 388-390.

8. Brockamp T, Nienaber $U$, Mutschler M, Wafaisade A, Peiniger S, Lefering $R$, et al. Predicting on-going hemorrhage and transfusion requirement after severe trauma: a validation of six scoring systems and algorithms on the TraumaRegister DGU ${ }^{\circledR}$. Crit Care Lond Engl. 20 juill 2012;16(4):R129.

9. Nunez TC, Voskresensky IV, Dossett LA, Shinall R, Dutton WD, Cotton BA. Early prediction of massive transfusion in trauma: simple as $A B C$ (assessment of blood consumption)? J Trauma. févr 2009;66(2):346-352.

10. Conway AM, Hinchliffe RF, Earland J, Anderson LM. Measurement of haemoglobin using single drops of skin puncture blood: is precision acceptable? J Clin Pathol. mars $1998 ; 51(3): 248-250$

11. Vincent JL. Lactate levels in critically ill patients. Acta Anaesthesiol Scand Suppl. $1995 ; 107: 261-266$

12. Baker SP, O'Neill B, Haddon W Jr, Long WB. The injury severity score: a method for describing patients with multiple injuries and evaluating emergency care. J Trauma. mars 1974;14(3):187-196. 
13. Husain FA, Martin MJ, Mullenix PS, Steele SR, Elliott DC. Serum lactate and base deficit as predictors of mortality and morbidity. Am J Surg. mai 2003;185(5):485-491.

14. Moomey CB Jr, Melton SM, Croce MA, Fabian TC, Proctor KG. Prognostic value of blood lactate, base deficit, and oxygen-derived variables in an LD50 model of penetrating trauma. Crit Care Med. janv 1999;27(1):154-161.

15. Choi JY, Lee WH, Yoo TK, Park I, Kim DW. A new severity predicting index for hemorrhagic shock using lactate concentration and peripheral perfusion in a rat model. Shock Augusta Ga. déc 2012;38(6):635-641.

16. Rixen D, Raum M, Holzgraefe B, Sauerland S, Nagelschmidt M, Neugebauer EA. A pig hemorrhagic shock model: oxygen debt and metabolic acidemia as indicators of severity. Shock Augusta Ga. sept 2001;16(3):239-244.

17. Manikis P, Jankowski S, Zhang H, Kahn RJ, Vincent JL. Correlation of serial blood lactate levels to organ failure and mortality after trauma. Am J Emerg Med. nov $1995 ; 13(6): 619-622$.

18. Régnier M-A, Raux M, Le Manach Y, Asencio Y, Gaillard J, Devilliers C, et al. Prognostic significance of blood lactate and lactate clearance in trauma patients. Anesthesiology. déc 2012;117(6):1276-1288.

19. Callaway DW, Shapiro NI, Donnino MW, Baker C, Rosen CL. Serum lactate and base deficit as predictors of mortality in normotensive elderly blunt trauma patients. J Trauma. avr 2009;66(4):1040-1044.

20. Paladino L, Sinert R, Wallace D, Anderson T, Yadav K, Zehtabchi S. The utility of base deficit and arterial lactate in differentiating major from minor injury in trauma patients with normal vital signs. Resuscitation. juin 2008;77(3):363-368.

21. Vandromme MJ, Griffin RL, Weinberg JA, Rue LW 3rd, Kerby JD. Lactate is a better predictor than systolic blood pressure for determining blood requirement and mortality: could prehospital measures improve trauma triage? J Am Coll Surg. mai 2010;210(5):861-867, 867-869.

22. Gaieski DF, Drumheller BC, Goyal M, Fuchs BD, Shofer FS, Zogby K. Accuracy of Handheld Point-of-Care Fingertip Lactate Measurement in the Emergency Department. West J Emerg Med. févr 2013;14(1):58-62.

23. Karon BS, Scott R, Burritt MF, Santrach PJ. Comparison of lactate values between 
point-of-care and central laboratory analyzers. Am J Clin Pathol. juill 2007;128(1):168-171.

24. Brinkert $\mathrm{W}$, Rommes $\mathrm{JH}$, Bakker J. Lactate measurements in critically ill patients with a hand-held analyser. Intensive Care Med. sept 1999;25(9):966-969.

25. Slomovitz BM, Lavery RF, Tortella BJ, Siegel JH, Bachl BL, Ciccone A. Validation of a hand-held lactate device in determination of blood lactate in critically injured patients. Crit Care Med. sept 1998;26(9):1523-1528.

26. Ferasin L, Nguyenba TP. Comparison of canine capillary and jugular venous blood lactate concentrations determined by use of an enzymatic-amperometric bedside system. Am J Vet Res. févr 2008;69(2):208-211.

27. Collange O, Charton A, Greib N, Joshi GP, Schaeffer R, Diemunsch PA. Correlation between arterial and capillary lactate measurements in a porcine hemorrhagic shock model. J Trauma. janv 2010;68(1):32-34.

28. Fauchère JC, Bauschatz AS, Arlettaz R, Zimmermann-Bär U, Bucher HU. Agreement between capillary and arterial lactate in the newborn. Acta Paediatr Oslo Nor 1992. 2002;91(1):78-81.

29. Frey B, Losa M. The value of capillary whole blood lactate for blood transfusion requirements in anaemia of prematurity. Intensive Care Med. janv 2001;27(1):222-227.

30. Coats TJ, Smith JE, Lockey D, Russell M. Early increases in blood lactate following injury. J R Army Med Corps. juin 2002;148(2):140-143.

31. Jansen TC, van Bommel J, Mulder PG, Rommes JH, Schieveld SJM, Bakker J. The prognostic value of blood lactate levels relative to that of vital signs in the pre-hospital setting: a pilot study. Crit Care Lond Engl. 2008;12(6):R160.

32. Hyacinthe A. Rapport d'activité du déchocage du CHU de Grenoble de 2007 à 2009. Mémoire de DES; 2011.

33. Patterson MJ, Galloway SD, Nimmo MA. Variations in regional sweat composition in normal human males. Exp Physiol. nov 2000;85(6):869-875.

34. Poortmans JR, Boisseau N. Biochimie des activités physiques. De Boeck Supérieur; 2003.

35. Van Beest PA, Mulder PJ, Oetomo SB, van den Broek B, Kuiper MA, Spronk PE. Measurement of lactate in a prehospital setting is related to outcome. Eur J Emerg Med Off J Eur Soc Emerg Med. déc 2009;16(6):318-322. 
36. Corradi F, Brusasco C, Garlaschi A, Santori G, Vezzani A, Moscatelli P, et al. Splenic Doppler resistive index for early detection of occult hemorrhagic shock after polytrauma in adult patients. Shock Augusta Ga. nov 2012;38(5):466-473. 


\section{Annexes}

Comparaison population de l'étude et population pour laquelle un lactate sanguin a été réalisé.

\begin{tabular}{|c|c|c|c|}
\hline $\begin{array}{c}\text { Variables quantitative } \\
\text { ou qualitatives }\end{array}$ & $\begin{array}{l}\text { Pas de lactate sanguin } \\
\qquad N=60\end{array}$ & $\begin{array}{l}\text { Lactate sanguin } \\
\qquad \mathrm{N}=60\end{array}$ & $p$ \\
\hline Age & 35 [31-56] & $41[26-54]$ & ns \\
\hline Sexe masculin & $53(88,3 \%)$ & $50(83,3 \%)$ & ns \\
\hline IMC & $23,1[22,2-25,6]$ & $24,2[21,9-25,2]$ & ns \\
\hline Grade & & & ns \\
\hline B & $20(33 \%)$ & 27 (45\%) & \\
\hline C & $40(67 \%)$ & $33(55 \%)$ & \\
\hline Temps pré-hospitalier (minutes) & $90[60-125]$ & 113 [79-150] & ns \\
\hline PAS PH & 118 [106-130] & 112.5 [98-135] & ns \\
\hline $\mathrm{FC} \mathrm{PH}$ & $81[72-94]$ & 79 [70-90] & ns \\
\hline Shock index & & & ns \\
\hline$<0,9$ & $42 / 58(72,4 \%)$ & $40 / 56(71,4 \%)$ & \\
\hline$>0,9$ & $16 / 58(37,6 \%)$ & $16 / 56(28,6)$ & \\
\hline Cristalloïde PH & $250[0-500]$ & $250[0-500]$ & ns \\
\hline Colloïde PH & 0 & 0 & ns \\
\hline Mécanisme lésionnel & & & ns \\
\hline compression & $1(1,7 \%)$ & $6(10 \%)$ & \\
\hline décélération & $52(86,7 \%)$ & $51(85 \%)$ & \\
\hline pénétrant & $7(11,6 \%)$ & $3(5 \%)$ & \\
\hline Hémocue & $13,6[12,6-14,6]$ & $13.6[12,3-15]$ & ns \\
\hline Score intensité clinique & $2[1-2]$ & $3[1,75-4]$ & $<<0,05$ \\
\hline
\end{tabular}




\begin{tabular}{|lccc|}
\hline Chirurgie d'hémostase DCA & $16(26,7 \%)$ & $11(18,3 \%)$ & $n s$ \\
Embolisation DCA & $2(3,3 \%)$ & $2(3,3 \%)$ & $n s$ \\
\hline ISS & $14[10-26]$ & $21[11-26]$ & $n s$ \\
SOFA & $0[0-1]$ & $1.5[0-4]$ & $p<0.05$ \\
SOFA CV & $0[0-0]$ & $0[0-4]$ & $p<0.05$ \\
\hline Transfusion $\geq 4 C G R$ à 48 h & $8(13,3 \%)$ & $4(6,7 \%)$ & $n s$ \\
\hline Hospitalisation en réanimation & $31(51,6 \%)$ & $21(35 \%)$ & $p<0.05$ \\
Infection en réanimation & $6 / 31(19,4 \%)$ & $17 / 21(28,3 \%)$ & $n s$ \\
Durée de séjour en réanimation & $5[3-11]$ & $8[4-18]$ & $p<0,05$ \\
DMS & $11[6-19]$ & $17[9-26]$ & $p<0,05$ \\
Mortalité à J28 & $1(1,7 \%)$ & $1(1,7 \%)$ & $n s$ \\
\hline
\end{tabular}

